\title{
Monadic constraint programming
}

\author{
TOM SCHRIJVERS \\ Department of Computer Science, K.U.Leuven, Leuven, Belgium \\ (e-mail: tom.schrijvers@cs.kuleuven.be) \\ PETER STUCKEY \\ National ICT Australia, Victoria Laboratory, Department of Computer Science \\ and Software Engineering, University of Melbourne, Melbourne, Australia \\ (e-mail: pjs@cs.mu.OZ.AU) \\ PHILIP WADLER \\ School of Informatics, University of Edinburgh, Edinburgh, Scotland, UK \\ (e-mail: philip.wadler@ed.ac.uk)
}

\begin{abstract}
A constraint programming system combines two essential components: a constraint solver and a search engine. The constraint solver reasons about satisfiability of conjunctions of constraints, and the search engine controls the search for solutions by iteratively exploring a disjunctive search tree defined by the constraint program. In this paper we give a monadic definition of constraint programming in which the solver is defined as a monad threaded through the monadic search tree. We are then able to define search and search strategies as first-class objects that can themselves be built or extended by composable search transformers. Search transformers give a powerful and unifying approach to viewing search in constraint programming, and the resulting constraint programming system is first class and extremely flexible.
\end{abstract}

\section{Introduction}

A constraint programming (CP; Marriott \& Stuckey, 1998) system combines two essential components: a constraint solver and a search engine. The constraint solver reasons about conjunctions of constraints, and its principal job is to determine unsatisfiability of a conjunction. The search engine controls the search for solutions by iteratively exploring an OR search tree defined by the program. Whenever the conjunction of constraints in one path defined by the search tree is unsatisfiable, search changes to explore another part of the search tree.

$\mathrm{CP}$ is a declarative programming formalism, where the constraints are defined declaratively, but the underlying constraint solvers are highly stateful, and indeed to specify complex search CP programs rely on reflecting state information from the solver. So in that sense CP is not so declarative after all.

In this paper we give a monadic definition of $\mathrm{CP}$ in which the solver is defined as a monad threaded through a monadic search tree. We are then able to define search and search strategies as first-class objects that can themselves be built or extended by composable search transformers. Search transformers give a powerful 


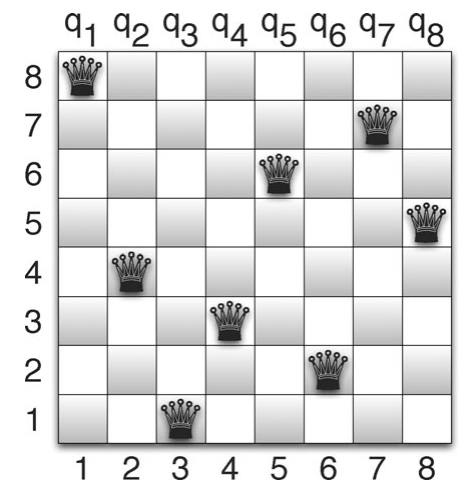

Fig. 1. A solution to the eight-queens problem.

and unifying approach to viewing search in CP. The resulting CP system is first class and extremely flexible.

Example 1. We use the well-known $n$-queens problem as a running example throughout this paper. The $n$-queens problem requires the placing of $n$ queens on an $n \times n$ chessboard, so that no queen can capture another. Since queens can move vertically, horizontally, and diagonally this means that

(1) no two queens share the same column;

(2) no two queens share the same row;

(3) no two queens share the same diagonal.

A standard model of the $n$-queens problem is as follows: Since we have $n$ queens to place in $n$ different columns, we are sure that there is exactly one queen in each column. We can thus denote the row position of the queen in column $i$ by the integer variable $q_{i}$. These variables are constrained to take values in the range $1 \ldots n$. This model automatically ensures the column constraint is satisfied. We can then express the row constraint as

$$
\forall 1 \leqslant i<j \leqslant n: q_{i} \neq q_{j}
$$

and the diagonal constraint as

$$
\forall 1 \leqslant i<j \leqslant n: q_{i} \neq q_{j}+(j-i) \wedge q_{j} \neq q_{i}+(j-i),
$$

since queens $i$ and $j$, with $i<j$, are on the same descending diagonal iff $q_{i}=$ $q_{j}+(j-i)$, and similarly they are on the same ascending diagonal iff $q_{j}=q_{i}+(j-i)$.

A solution to the eight-queens problem is shown in Figure 1. The solution illustrated has $q_{1}=8, q_{2}=4, q_{3}=1, q_{4}=3, q_{5}=6, q_{6}=2, q_{7}=7, q_{8}=5$.

The first role of a CP language is to be able to succinctly model problems. We will define CP in Haskell that allows the model of the $n$-queens problem shown in Figure 2. Note how similar it is to the mathematical model.

The next important part of a CP solution is to be able to program the search. We will construct a language for search that allows us to express complex search strategies succinctly - and in a composable manner. 


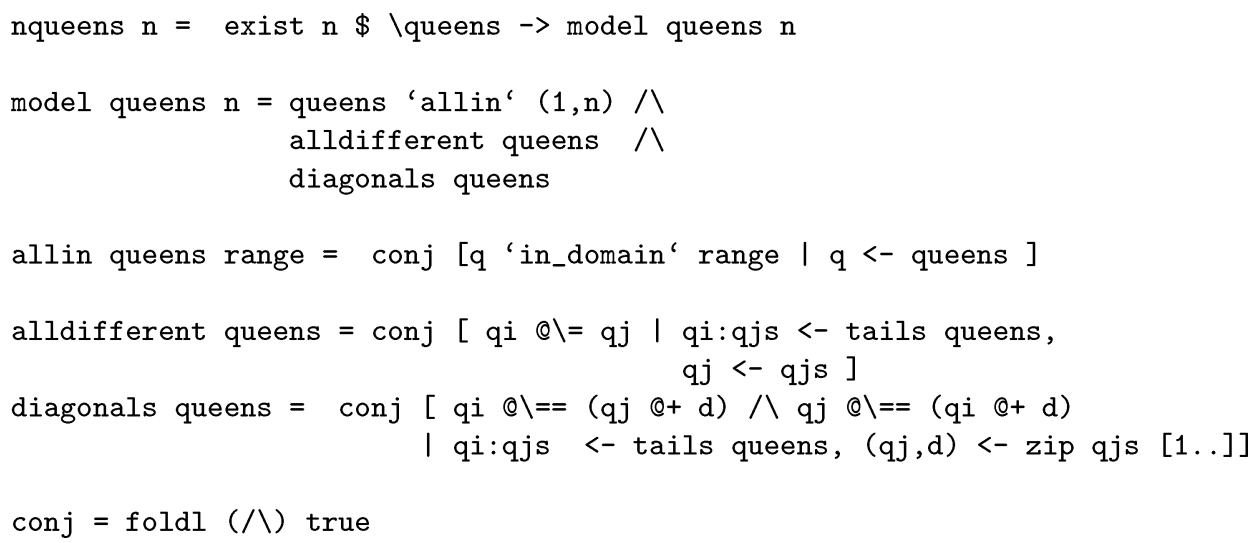

Fig. 2. Haskell code for modeling $n$ queens.

Search is separated into components: specifying the search tree, the basic order for visiting the search tree and then the search transformers that transform the search tree or the way it is visited. Examples of search orders are depth-first search (dfs), breadth-first search (bfs) and best-first search (BeFS). Examples of search transformers are depth-bounded search ( $\mathrm{db} n$ never visits nodes at depth below $n$ ), node-bounded search ( $\mathrm{nb} n$ visits at most $n$ nodes), limited-discrepancy search (ld $n$ visits only nodes requiring at most $n$ right branches), and branch-and-bound optimization ( $\mathrm{bb} f$ applies a tree transformation $f$ for eliminating nonoptimal solutions). These search transformers are composable, so we can apply multiple transformations in order.

Example 2. For example, using our search framework we can succinctly define complex search strategies. The following calls show how to solve eight queens with

- depth-first search (DFS), first applying a node bound of 100, then a depth bound of 25, then using newBound branch and bound;

- breadth-first search (BFS), first applying a depth bound of 25, then a node bound of 100, then using newBound branch and bound;

- BFS, first limited discrepancy of 10 , then a node bound of 100 , then using newBound branch and bound

The above can be expressed in our framework as

$$
\begin{aligned}
& >\text { solve dfs (nb } 100:- \text { db } 25:- \text { bb newBound) } \$ \text { nqueens } 8 \\
& >\text { solve bfs (db } 25:- \text { nb } 100:- \text { bb newBound) } \$ \text { nqueens } 8 \\
& >\text { solve bfs (ld } 10:- \text { nb } 100:- \text { bb newBound) } \$ \text { nqueens } 8
\end{aligned}
$$

Clearly exploring different search strategies is very straightforward.

We hope that his paper will illustrate to the functional programming community how the abstractions and mechanisms from functional programming such as monads, higher-order functions, continuations, and lazy evaluation are valuable notions for defining and building CP systems. 
Functional abstractions have been used throughout the development of CP. Indeed in the evolution of $\mathrm{CP}$ we have seen a series of increasingly complex abstractions as the field became better understood. The original CLP(X) framework (Jaffar \& Lassez, 1987) already abstracted the constraint language and solver from the rest of the language. Constraint logic programming (CLP) languages such as ECLIPSE (ECLiPSe, 2008) provided search abstractions that allowed features of search such as variable and value selection to be defined by users code. The Oz (Smolka, 1995) language separated the definition of the search tree, from the method used to explore it, providing functional abstractions for defining the search. OPL (Van Hentenryck et al., 2000) provided for the first time search strategy transformers in the form of search limiters and mechanisms to define these transformers. Most recently Comet (Van Hentenryck \& Michel, 2006) defined search through continuations and provided functional abstractions to thread complex state through a search in order to build complex search strategies.

Our work can be viewed as encapsulating the functional abstractions previously used in $\mathrm{CP}$ in a functional programming language and as using the power of functional programming to take a further step in the increasingly abstract view of search and CP. The following are the contributions of this paper:

- We show how monads provide a powerful tool for implementing CP abstractions, which allows us to build a highly generic framework for CP.

- We define search strategy transformers that are composable transformers of search and show how we can understand existing search strategies as constructed from more fundamental transformers.

- We open up a huge space of exploration for search transformers.

The code is available at http://www.cs.kuleuven.be/ toms/Haskell/.

The remainder of the paper is organized as follows: In Section 2 we give a generic definition of a constraint solver and show how it can be instantiated by a simple finite domain (FD) solver. In Section 3 we define a structure for representing conjunctive constraint models. In Section 4 we extend the structure to model disjunctive constraint models and now have sufficient expressiveness to run a model for the first time. In Section 5 we extend the modeling structure to allow dynamic definitions of the model and are now able to define a basic CP system, with user-defined search. In Section 6 we introduce basic search strategies that define the order in which nodes in the dynamic search tree are visited. In Section 7 we introduce search strategy transformers, which allow us to modify a given search as it proceeds. In Section 8 we extend search strategy transformers to be composable, so that we can apply multiple transformations to an underlying search. In Section 9 we discuss related work, and in Section 10 we conclude and discuss future work.

\section{Constraint solvers}

The core component of a CP system is the constraint solver. This is the engine that determines if conjunctions of constraints may be satisfiable and that can reflect information that it knows about a conjunction of constraints. 
We define a fully generic constraint solver interface in what follows, and then we show how this interface can be instantiated for our FD constraint solver for $n$ queens.

\subsection{Generic solver interface}

Our generic constraint solver interface is captured by the Solver type class:

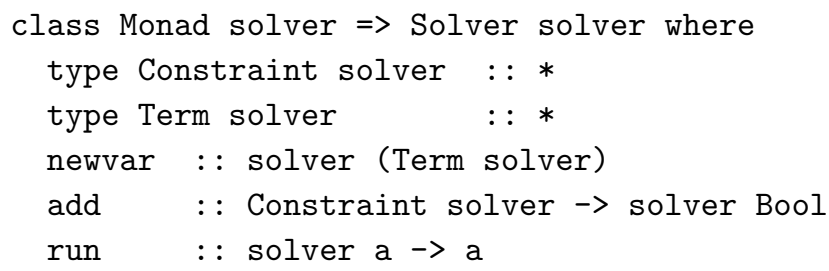

This type class requires that solver be a monad, which allows it to encapsulate its state. A solver has two associated types (Schrijvers et al., 2008), the type of supported constraints (Constraint solver) and the type of terms that constraints range over (Term solver).

The terms of interest are of course the constraint variables; a fresh variable is generated by the newvar function. Constraints over terms are added to the solver's current set of constraints (its state) by the add function. The function also returns whether this addition leads to a possibly consistent set of constraints - it returns False if the solver knows that the conjunction of constraints is inconsistent. As this is the only operation that may fail, it is undesirable to require that solver is an error monad. Resorting to a monad transformer to indicate the potential failure of add, e.g., by giving it signature Constraint solver $\rightarrow$ MaybeT solver (), strikes us as overkill too.

Finally, the run function allows us to run an action in the solver monad and get back a result.

\subsection{A simple $F D$ solver}

To illustrate the above generic solver interface, we now present a simple instantiation, without going into the implementation details.

Our solver type is called FD and its instance of the Solver class is

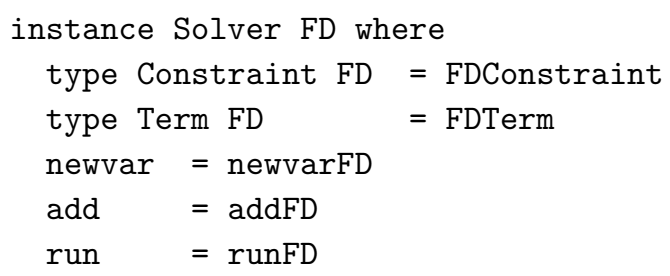

The FDTerm type is abstract, and of course the details of the member functions are not exposed. All the programmer needs to know are the details of the FDConstraint type. Our small FD solver only supports three constraints:

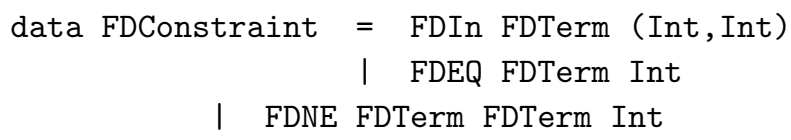


The first, FDIn, restricts a variable to a range; the second, FDEQ, forces a variable to take a value; and the third, FDNE, expresses that a variable is not equal to the sum of another variable and an integer. Formally, the semantics can be expressed as

$$
\begin{aligned}
\llbracket \text { FDIn } t(l, u) \rrbracket & =\llbracket t \rrbracket \in\{l, \ldots, u\} \\
\llbracket \text { FDEQ } t d \rrbracket & =\llbracket t \rrbracket=d \\
\llbracket \text { FDNE } s \text { t } i \rrbracket & =\llbracket s \rrbracket \neq \llbracket t \rrbracket+i
\end{aligned}
$$

We use Overton's FD solver (Overton, 2008) for the concrete implementation.

The above is sufficient to express our $n$-queens model. However, it is a rather primitive way of writing a model, directly against the constraint solver interface. In the next section, we define a more suitable generic model abstraction that is further away from the constraint solver interface.

Note that global constraints, such as alldifferent (Régin, 1994), an important feature of $\mathrm{CP}$, may be supported directly by the solver by making them part of the Constraint solver type. They can also be defined succinctly by decomposition to more primitive constraints (as we do for alldifferent in Figure 2) using the primitive solver interface.

\section{Model tree}

We wish to represent the constraint model as a separate data type rather than a composition of functions from the constraint solver interface. This has many obvious advantages for manipulating and inspecting the model, which will be handy later on.

Although right now our model is only a heterogeneous sequence, we call the model data type Tree:

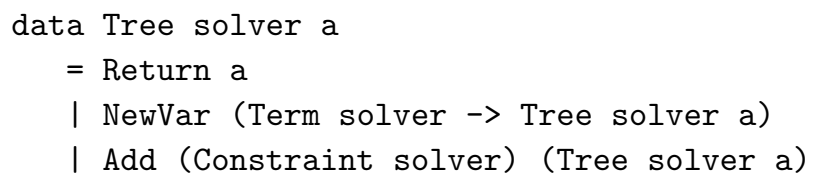

This data type has the two obvious constructors NewVar and Add that mimic the corresponding functions from the solver interface. Finally, the Return constructor is the base case and marks the end of a model.

The type Tree has two type parameters: solver is the obvious constraint solver type, while a is a value returned in the Return node. It turns the Tree type into its own free monad:

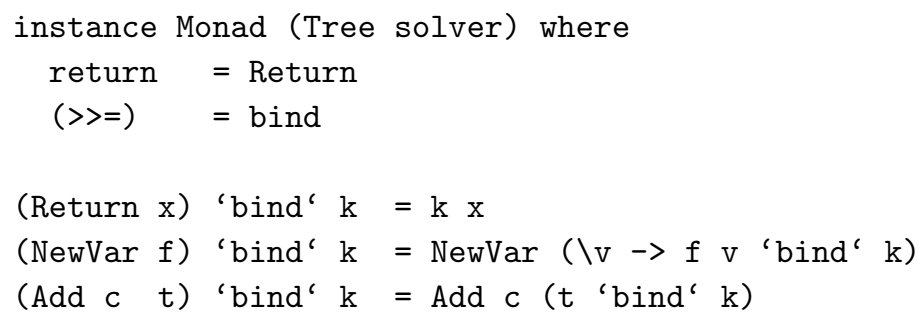




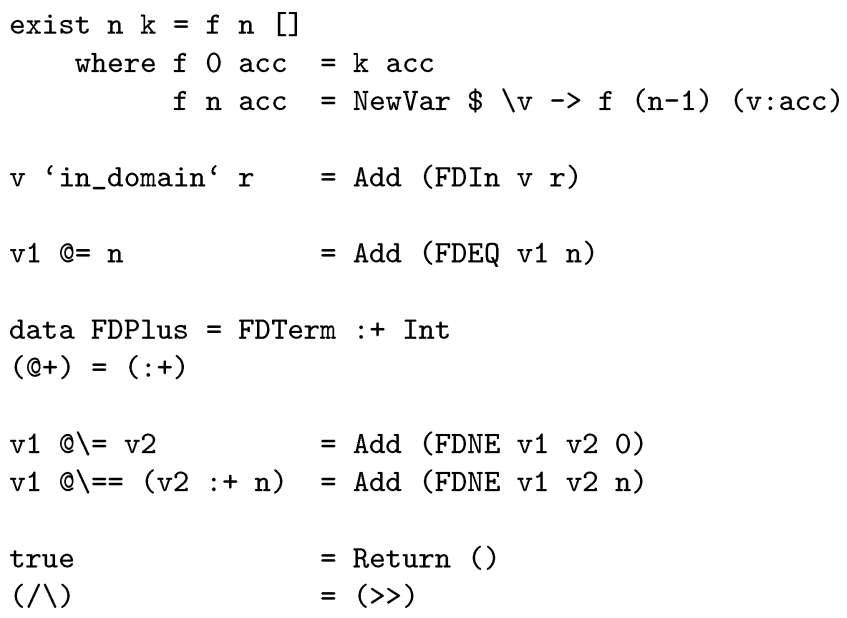

Fig. 3. Solver interface as Tree generator for $n$ queens.

Observe that bind effectively extends a model by replacing the base case Return by another model.

In terms of this new model data type, we can express the auxiliary functions of the $n$-queens problem as well. They are shown in Figure 3. It is seen that exist $n$ $k$ creates $n$ new variables passed as an argument to $k$; $v$ in_domain $r$ constrains variable $v$ to be in range $r ; @=, \bigotimes \backslash=$, and $@ \backslash==$ are syntactic sugar for the underlying constraint of equality, disequality, and disequality with offset; true represents the always true constraint; and conjunction $\wedge$ is simply the monadic $>>$ bind operation.

Because the model itself now does not run the problem through the solver, we must provide a separate "evaluation" function:

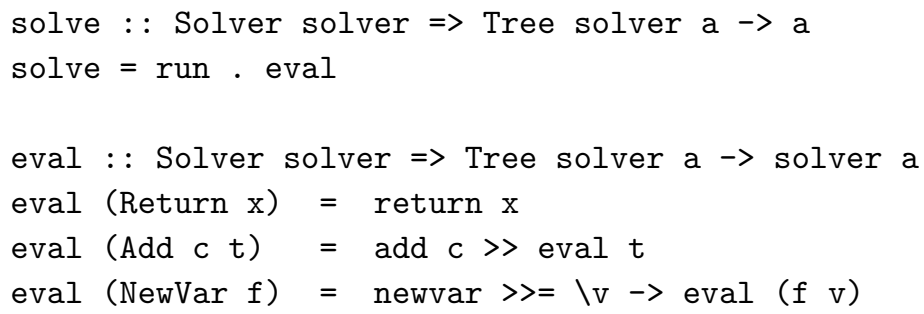

\section{Disjunctive model tree}

FD solvers, as well as most other constraint solvers, are incomplete; that is, when given a conjunction of constraints they can give three possible answers: satisfiable, unsatisfiable, or unknown (when the solver cannot determine if the conjunction is satisfiable or not). In order to complete a constraint solver and find one or more solutions, additional constraints must be added until the solver establishes a solution - or inconsistency. Of course, it is not a priori known what constraints need to be added in order to find a solution; one must try out different alternatives. 
For this purpose we extend the model tree data type with two new constructors:

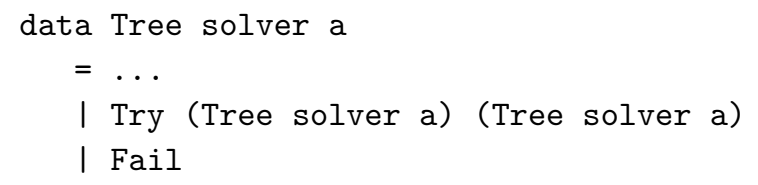

The Try constructor denotes two disjoint alternatives, for exploring the space of candidate solutions. The Fail constructor denotes a dead end. The bind function is extended in the obvious manner to cover these new constructors:

$$
\begin{aligned}
& \text { Fail } \text { 'bind' } \mathrm{k}=\text { Fail } \\
&(\operatorname{Try} 1 \mathrm{r} \text { ) 'bind' } \mathrm{k}=\operatorname{Try}(\mathrm{l} \text { 'bind' } \mathrm{k}) \text { ( } \mathrm{r} \text { 'bind' } \mathrm{k})
\end{aligned}
$$

Note that the binary Try constructor could be generalized to an arbitrary number of alternatives, giving it the signature Try :: [Tree solver a] $\rightarrow$ Tree solver a. For the simplicity of the presentation, we avoid doing so. Moreover, no expressiveness is lost, as variadic disjunctions are easily decomposed into binary disjunctions.

\subsection{Solving with branches: Solver state snapshots}

We need to extend our solver to handle Try nodes. In order to do so we make a minimal extension to the constraint solver interface:

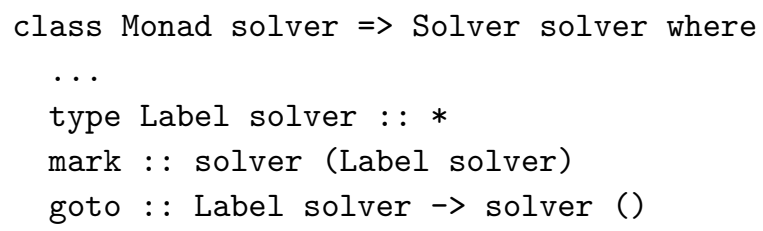

The solver must now support a "label" type, which represents a solver state in one way or another. The mark function returns a label for the current solver state, and the goto function brings the solver back into an earlier state.

It is up to the solver to choose a representation for its labels and what strategy to use for returning to a previous state from the current one. Two common techniques are copying and trailing. In the former approach, a label is a copy of the whole solver state and the two functions are obvious. A copying solver could also use adaptive recomputation (Schulte, 1999).

In the latter approach, a label is a trail of incremental changes. Navigating from the current state to a previous state happens as follows:

- We determine all common incremental changes between the trails of the two states, starting at the root.

- All other changes of the current state are undone.

- All other changes of the previous state are redone.

The idea is that the incremental changes are cheaper to undo and redo than actual full recomputation. 


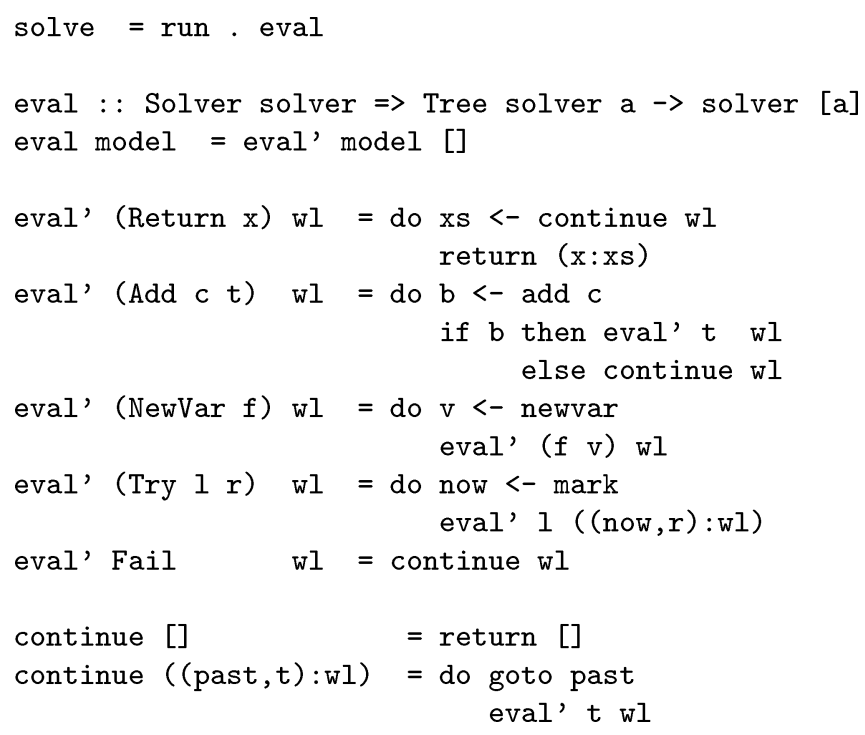

Fig. 4. Code for solve showing how a model tree is evaluated by threading a solver along its branches.

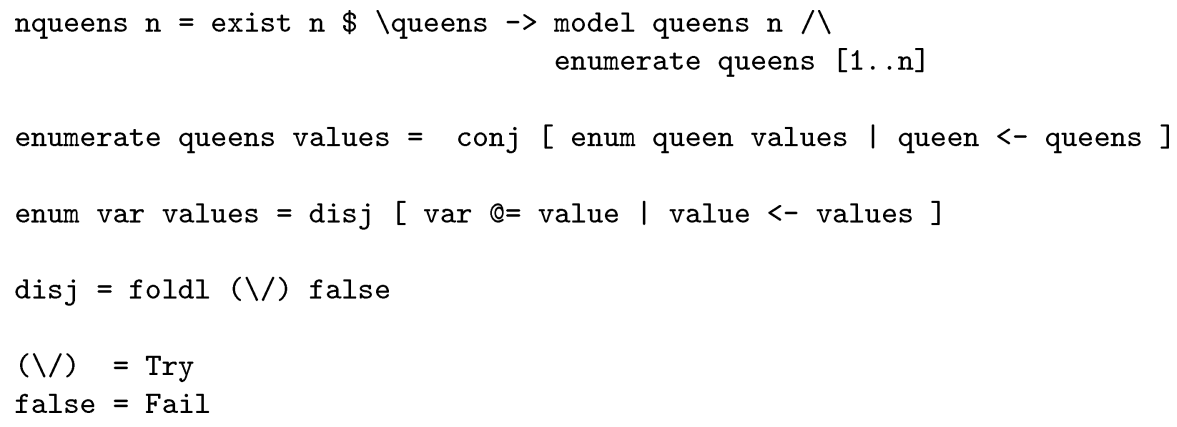

Fig. 5. Model for $n$ queens with explicit enumeration of variables values.

Now the solving strategy goes down one branch but remembers to revisit the other branch: the branch is pushed onto a worklist together with a label for the current solver state. The code for solve is shown in Figure 4. Here the worklist is a stack and the search is implicitly depth-first left to right.

Example 3. With these new constructors, we can now extend the $n$-queens model to enumerate the possible values of each variable in different disjunctions. The resulting code is shown in Figure 5. The enumerate function creates a conjunction of enumerations using enum var values that creates a disjunction setting the variable to each of the given values. Note how disjunction $\backslash$ is simply Try and the false constraint is Fail.

The enumerate function constructs a tree of disjunctive constraints; for example, for two queens it constructs the tree shown in Figure 6, which is represented by the term 


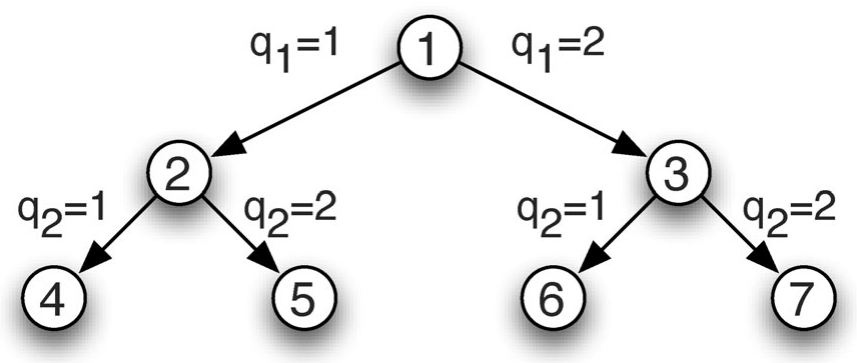

Fig. 6. Enumeration tree for two queens.

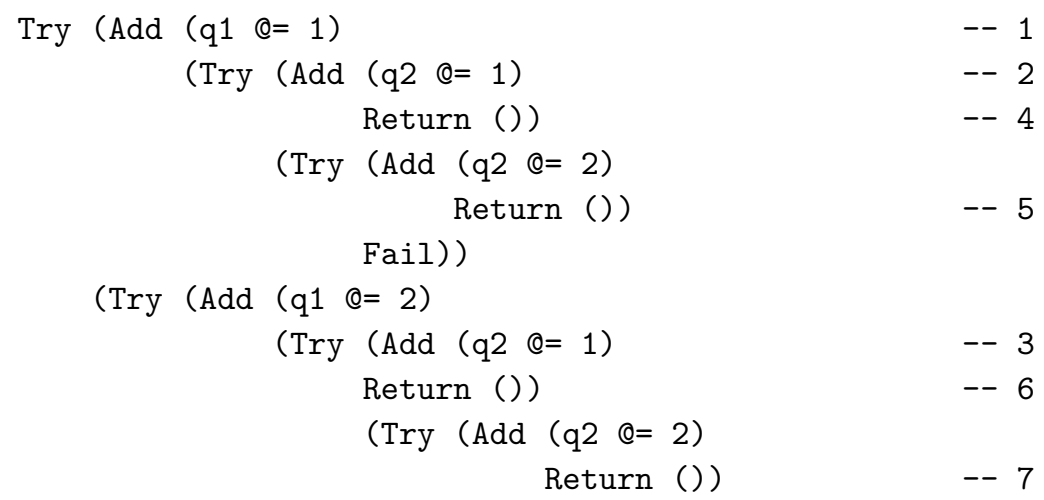$$
--2
$$

We can now run the code:

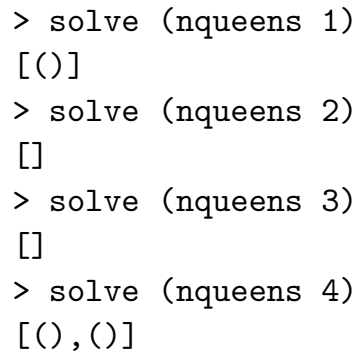

Each () indicates a solution.

As the example above shows, running the solver is not very informative. The result tells us there are two solutions but does not tell us what they actually are. Yet, what we are really interested in are of course the actual solutions.

One way to return the actual solutions is to modify the enumerate function and make it generate a Return leaf that lists the assignments made. However, in the next section we will see a much more concise way to achieve the same effect. 


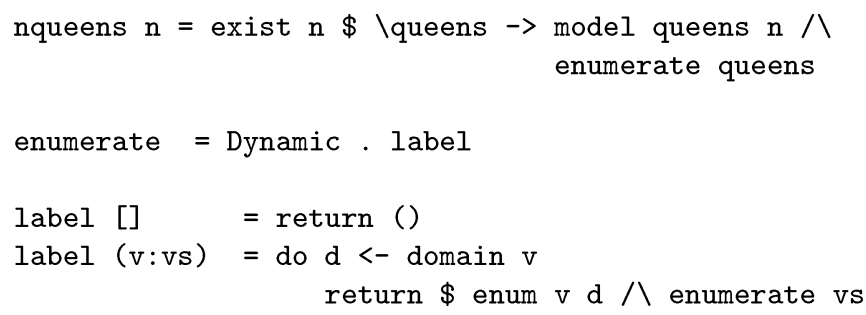

Fig. 7. Code for enumerating the search for $n$ queens.

\section{Dynamic model tree}

The full $n$-queens model tree has an overwhelming $n^{n}$ leaves as well as a proportional number of internal Try nodes. Even though lazy evaluation will avoid constructing all of this tree, many of the choices immediately lead to failure. We can do much more efficient and interesting search if we can make use of dynamic information in the solver.

In this section, we show several techniques for dynamically building a smaller tree, based on the solver state. Importantly we only build the tree for the parts of the search space we actually explore. For this purpose, we add one more constructor to the tree data type:

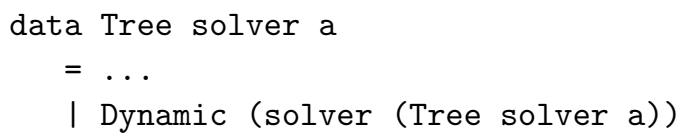

The evaluation function deals with this new constructor as follows:

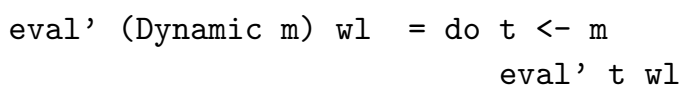

Note that the Dynamic m adds additional expressiveness because the generated tree is not fixed statically but depends on the dynamic solver state.

\subsection{Branching on the dynamic range}

Earlier, we branched the $n$-queens model on the full static range $(1, n)$ of each variable. However, many of the values in this range will directly result in inconsistency. Indeed, FD constraint solvers keep track of each variable's set of possible values. This domain dynamically shrinks as new constraints are added. By the time that possible values are enumerated a queen's domain has shrunk to a set $d \subseteq\{1 . . n\}$. Any attempt to assign a value $v$, where $v \notin d$, is in vain. By dynamically inspecting the variable's current domain, we can avoid generating these redundant attempts.

Example 4. We assume that the FD constraint solver exposes a function domain $:$ : FDTerm $\rightarrow$ FD [Int] to inspect a variable's current domain. Then we can dynamically generate the enumeration subtree, based on each variable's dynamic domain. The code is shown in Figure 7. 


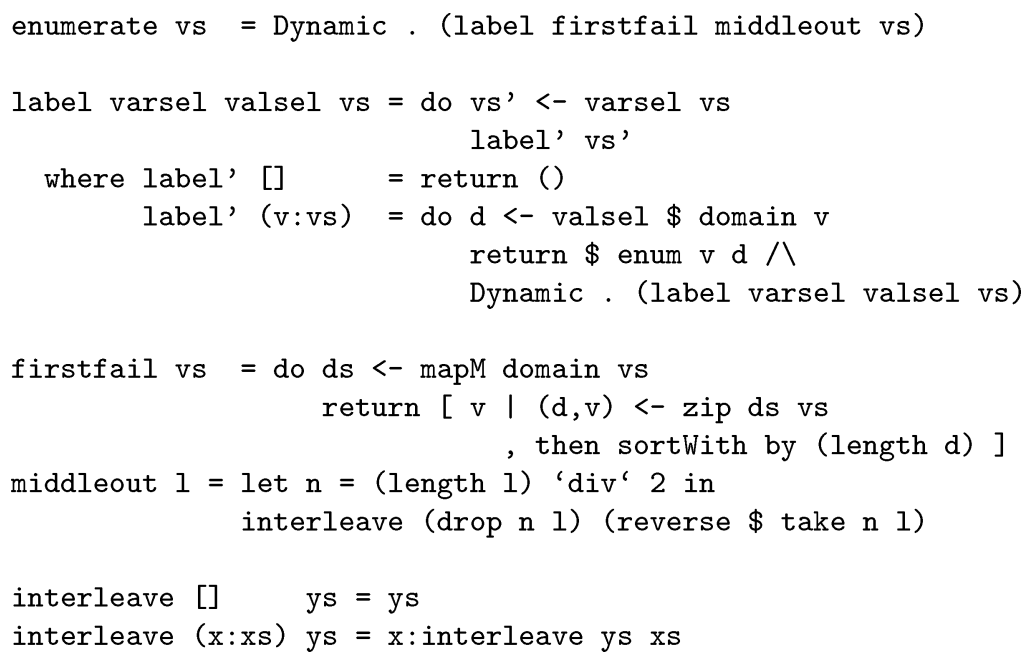

Fig. 8. Code for generic labeling predicate and examples of first-fail variable selection and middleout value ordering.

\subsection{Variable and value selection}

So far we have selected the queen variables for value assignment in their natural order. However, there is no particular semantic reason to do so: any order yields the same solutions, though perhaps in a different order. However, the size of the search tree may be quite different. It turns out that for $n$ queens we get a much smaller search tree, if we select the variable with the smallest domain first; this is called the first-fail principle. The idea behind first-fail is that straining the bottleneck, the variable with the smallest degree of freedom, exposes inconsistency more quickly.

Similarly we can order the way in which we try values for each queen. This does not change the size of the search tree but may push the solutions to the left of the search tree, so in left-to-right search they are found earlier. For the $n$-queens problem it is known that trying the values of a variable from the middle of the board outwards is beneficial.

Figure 8 gives generic code for a labeling function that takes two arguments: varsel reorders a list of variables so that the selected variable is first, while valsel reorders a list of values so that they are tried in the left-to-right order. The figure also shows implementations of first-fail variable selection and middleout value ordering. First-fail variable selection chooses the variable with least domain size. Middleout ordering tries values closest to the middle of the domain first, implemented by splitting the list in half and interleaving the reverse of the first half with the second half.

\subsection{Running the solver revisited}

If the FD solver exposes a function value : : FDTerm $\rightarrow$ FD Int that returns the value assignment of a variable, the Dynamic constructor allows us to return the solution in a highly convenient way: 


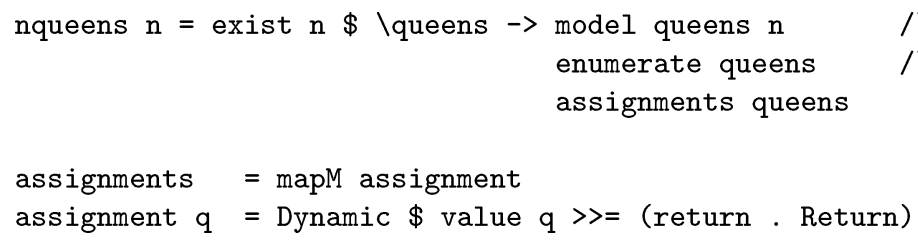

Fig. 9. Code to output the solutions to an $n$-queens problem.
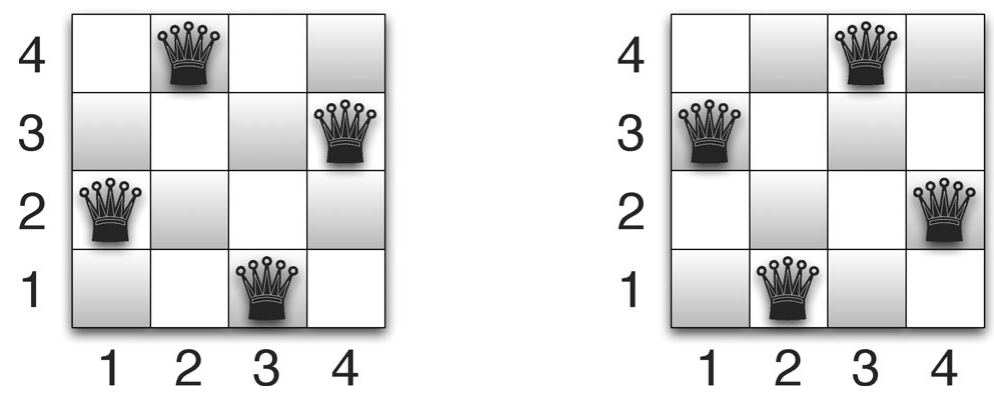

Fig. 10. The two solutions to the four-queens problem.

Example 5. Let's extend our $n$-queens code, so that the solutions are returned. We simply need to capture the assignments of the variables at a solution and return them. The code is shown in Figure 9. Now, running the solver, we get to see the actual solutions:

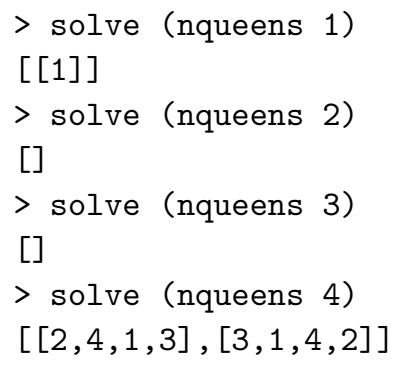

The two solutions to the four queens problem are shown in Figure 10.

\subsection{Alternate labeling strategies}

Being able to flexibly define a dynamic model tree is a key capability for CP. To show the importance of the right labeling approach we compare some different approaches.

Table 1 compares the number of nodes visited to find all solutions, or just the first solution, for four different strategies:

- in order, the default approach defined in Figure 7;

- first fail (ff) using the code of Figure 8 with enumerate = Dynamic . (label firstfail id);

- $\mathrm{ff}+$ middle out using the code of Figure 8; and

- ends out where variables are selected alternately from each end of the board (a bad search order). 
Table 1. Table comparing the number of 'Try' nodes visited to find 'All' or the 'First' solution, using various labeling strategies; - indicates more than 100,000 nodes

\begin{tabular}{|c|c|c|c|c|c|c|c|c|}
\hline \multirow[b]{2}{*}{$n$} & \multicolumn{2}{|c|}{ in order } & \multicolumn{2}{|c|}{ first fail } & \multicolumn{2}{|c|}{$\mathrm{ff}+$ middle out } & \multicolumn{2}{|c|}{ ends out } \\
\hline & All & First & All & First & All & First & All & First \\
\hline 1 & 9 & 8 & 9 & 8 & 9 & 8 & 9 & 8 \\
\hline 2 & 13 & 13 & 13 & 13 & 13 & 13 & 13 & 13 \\
\hline 3 & 23 & 23 & 23 & 23 & 23 & 23 & 23 & 23 \\
\hline 4 & 84 & 52 & 84 & 52 & 84 & 44 & 84 & 52 \\
\hline 5 & 282 & 62 & 280 & 62 & 280 & 62 & 288 & 64 \\
\hline 6 & 329 & 117 & 325 & 113 & 325 & 103 & 369 & 139 \\
\hline 7 & 1,531 & 107 & 1,479 & 107 & 1,479 & 109 & 1,683 & 129 \\
\hline 8 & 4,378 & 254 & 4,286 & 252 & 4,286 & 138 & 5,166 & 228 \\
\hline 9 & 17,496 & 190 & 16,756 & 186 & 16,756 & 166 & 21,318 & 290 \\
\hline 10 & 54,141 & 285 & 52,371 & 221 & 52,371 & 205 & 73,515 & 423 \\
\hline 11 & - & 261 & - & 293 & - & 259 & - & 839 \\
\hline 12 & - & 514 & - & 494 & - & 276 & - & 566 \\
\hline 13 & - & 370 & - & 544 & - & 354 & - & 1,848 \\
\hline 14 & - & 1,995 & - & 501 & - & 375 & - & 2,487 \\
\hline 15 & - & 1,555 & - & 421 & - & 473 & - & 4,465 \\
\hline 16 & - & 9,738 & - & 480 & - & 518 & - & 33,418 \\
\hline 17 & - & 6,430 & - & 584 & - & 544 & - & 17,070 \\
\hline 18 & - & 37,797 & - & 665 & - & 683 & - & - \\
\hline 19 & - & 3,631 & - & 761 & - & 647 & - & - \\
\hline 20 & - & - & - & 748 & - & 830 & - & - \\
\hline 21 & - & - & - & 954 & - & 1,358 & - & - \\
\hline 22 & - & - & - & 845 & - & 861 & - & - \\
\hline 23 & - & - & - & 901 & - & 905 & - & - \\
\hline 24 & - & - & - & 1,008 & - & 996 & - & - \\
\hline 25 & - & - & - & 1,082 & - & 1,534 & - & - \\
\hline 26 & - & - & - & 1,137 & - & 1,195 & - & - \\
\hline 27 & - & - & - & 1,219 & - & 1,251 & - & - \\
\hline 28 & - & - & - & 1,536 & - & 1,362 & - & - \\
\hline 29 & - & - & - & 1,664 & - & 1,532 & - & - \\
\hline 30 & - & - & - & 1,893 & - & 1,525 & - & - \\
\hline 31 & - & - & - & 1,679 & - & 1,817 & - & - \\
\hline 32 & - & - & - & 1,800 & - & 3,390 & - & - \\
\hline 33 & - & - & - & 5,670 & - & 2,060 & - & - \\
\hline 34 & - & - & - & 2,081 & - & 2,529 & - & - \\
\hline 35 & - & - & - & 2,309 & - & 2,057 & - & - \\
\hline 36 & - & - & - & 2,194 & - & 2,610 & - & - \\
\hline 37 & - & - & - & 2,372 & - & 2,272 & - & - \\
\hline 38 & - & - & - & 2,443 & - & 2,985 & - & - \\
\hline 39 & - & - & - & 2,745 & - & 2,655 & - & - \\
\hline 40 & - & - & - & 3,492 & - & 2,608 & - & - \\
\hline
\end{tabular}

Clearly first fail significantly improves upon in order, while ff + middle out usually improves again for finding the first solution (for all solutions it is identical to first fail). The ends out search approach is very bad. 


\section{Search strategies}

The way our evaluation function is implemented is through visiting the nodes in the tree in a depth-first order, i.e., realizing DFS. DFS is not always the best search strategy if we want to get the first solution as quickly as possible. For instance, if the solution resides far to the right in the tree, DFS will find it late in the search. Then BFS may be a better choice. Another approach is BeFS that orders the nodes to visit based on a heuristically determined priority.

It is common folklore that all of these search strategies are instances of a single primitive search strategy that is parametric in the queuing data type. We capture the generic queuing data type interface in the Queue type class:

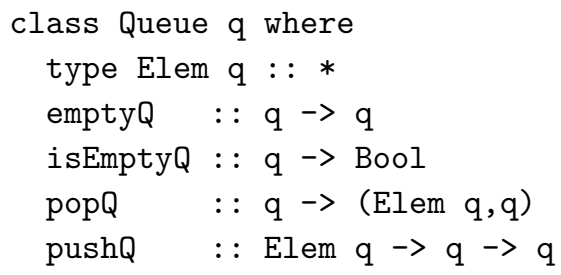

Here Elem $\mathrm{q}$ is the type of the elements in the queue, and the obvious functions are supported.

The evaluation function is adapted to use this Queue interface:

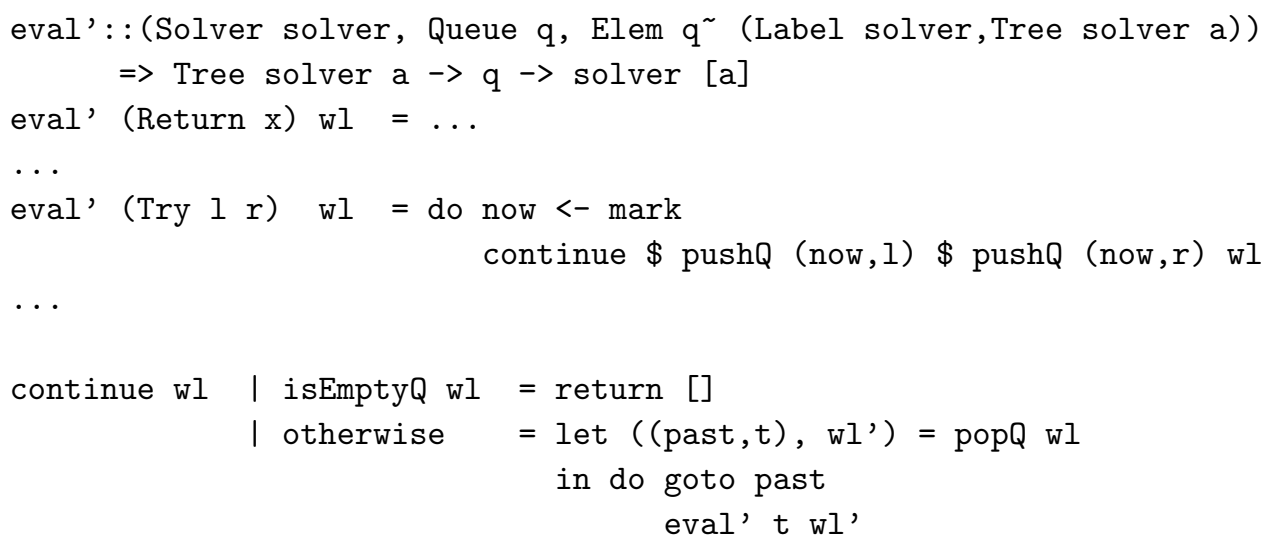

By choosing a LIFO queue (a stack), a FIFO queue, or a priority queue we obtain respectively DFS, BFS, and BeFS:

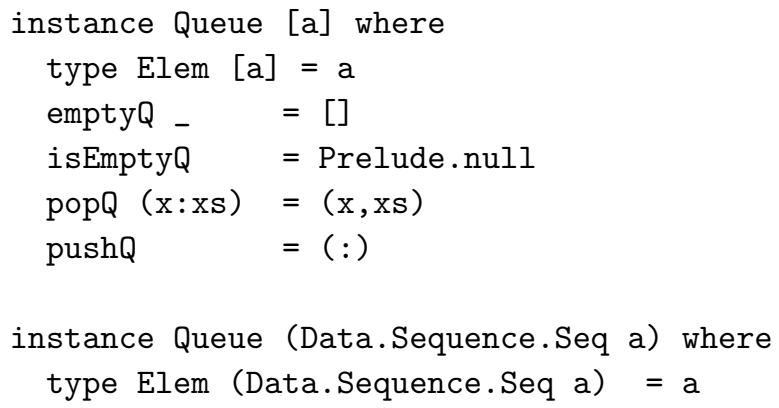




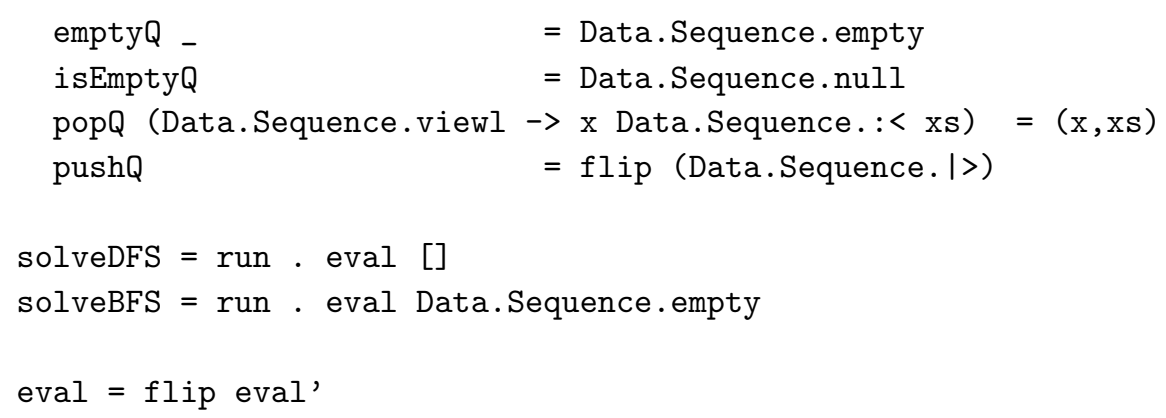

\section{Search strategy transformers}

Many more search strategies can be expressed naturally in terms of transformations of the above primitive queue-based search strategy. For example, node-bounded search only explores a certain number of nodes; depth-bounded search only explores nodes up to a certain depth; and limited-discrepancy limits the number of right branches.

Hence, we introduce a type class of SearchTransformers:

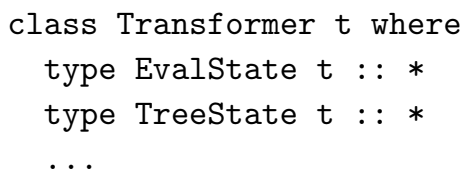

A search transformer $t$ has its own state to base decisions for steering the search on. This state is composed of two separate parts: the EvalState $t$ is threaded through the evaluation from one visited tree node to the next, while the TreeState $t$ is threaded top-down through the tree from a parent tree node to its children. The usefulness of these two state components will become clear when we consider specific search transformer instances.

The search transformer acts as an extra layer on top of the primitive search. Whenever a new node is selected for processing, the search transformer gets to see it first, and when it's done, the transformer delegates the node to the primitive search.

This means that we have to modify the code of the evaluation function to carry around the transformer and its state and to call the transformer at the appropriate times:

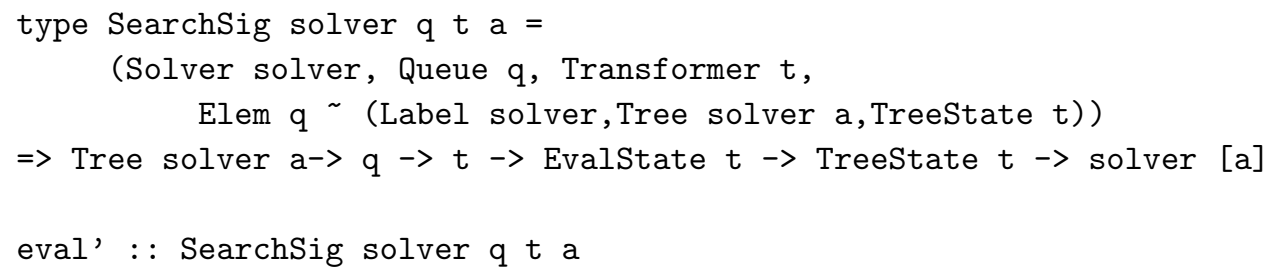

The two fundamental changes to the code are in the Try case of eval' and in continue:

eval' ( $\operatorname{Try} 1 \mathrm{r}$ ) wl $\mathrm{t}$ es $\mathrm{ts}=$

do now <- mark 


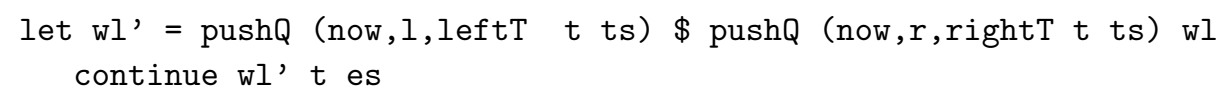

The new functions leftT, rightT, and nextT are methods of the Transformer type class:

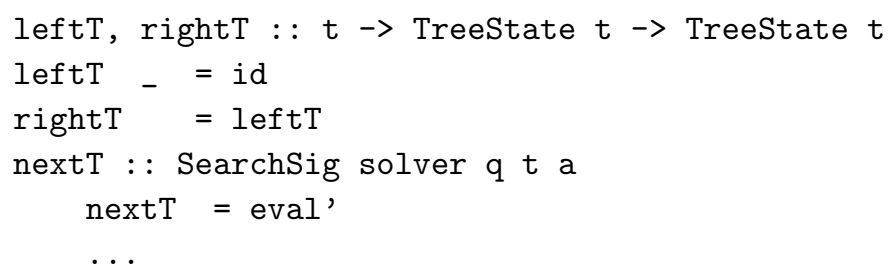

With leftT and rightT, the transformer specifies how the tree state is inherited from a Try node. The default implementation for the right child does the same as the left child, and the latter simply copies the parent's node state.

The nextT method is a proxy for eval', and by default it simply calls eval'. Note that they have the same signature.

In order to start a new search, we provide the main evaluation function

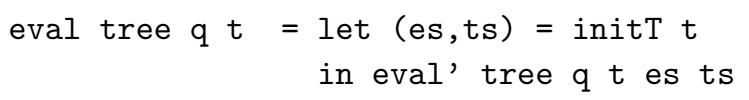

that uses the last method of the Transformer class, for initializing the global and node states:

$$
\text { initT }:: t \rightarrow \text { (EvalState } t, \text { TreeState } t \text { ) }
$$

\subsection{Transformer implementations}

Now that we have the infrastructure, let us look at a few concrete search transformers.

Depth-bounded search transformer. In depth-bounded search, we do not explore any nodes beyond a certain depth in the tree. For this purpose, the tree state represents the depth of the node and the eval state simply records if any depth pruning occurred (which will be useful later):

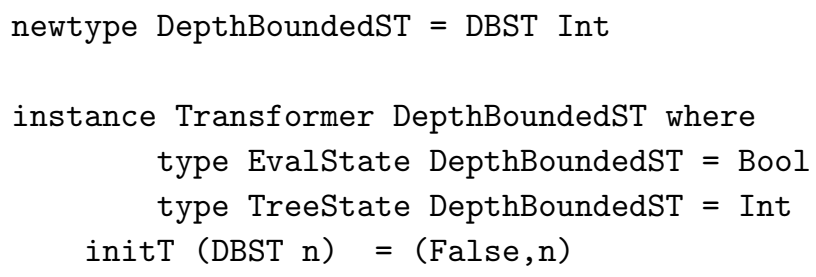




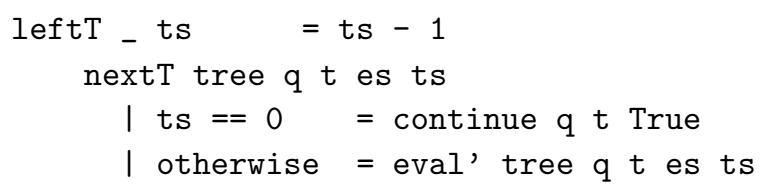

The initial depth limit is embedded in the DepthBoundedST value. We see in leftT that each time a left (and through defaulting also right) branch is taken, the limit decreases. When the limit hits 0 , then nextT does not continue evaluation at the current node and changes the eval state to True to indicate pruning. This effectively cuts off the model tree at the given depth, fully orthogonal to the queuing strategy.

Node-bounded search transformer. In node-bounded search, at most $n$ nodes in the tree are explored. Hence, its eval state Int denotes the number of remaining nodes, and the tree state is unused ${ }^{1}$ :

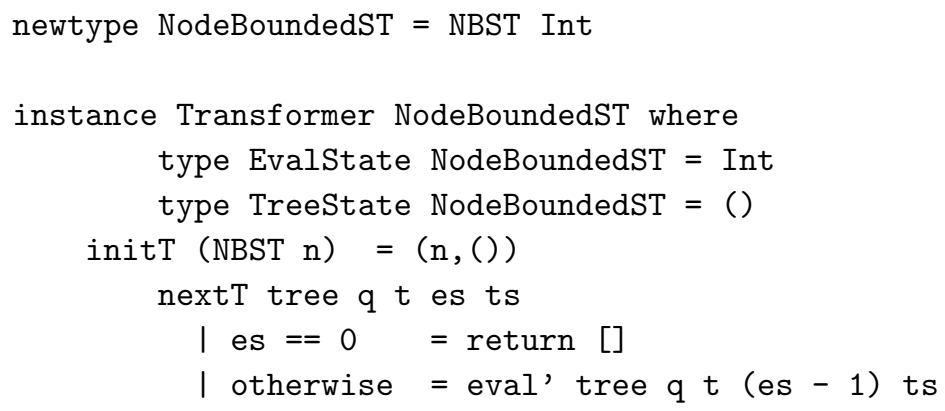

The implementation of the node-bounded search transformer is almost identical to that of the depth-bounded search transformer. However, the subtle interchange of the roles of the two states has quite a different impact on where the tree is cut off. Unlike the previous transformer, the impact of the current transformer is sensitive to the underlying queuing strategy.

Limited-discrepancy search transformer. In limited-discrepancy search (LDS), the leftmost path in the tree is visited, and any other path that deviates at most $n$ alternatives from the leftmost path:

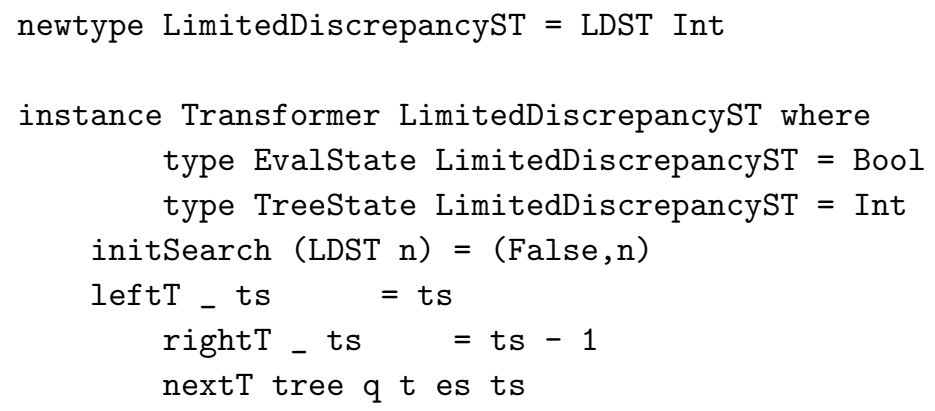

1 We use Haskell's unit type () to denote empty state. 

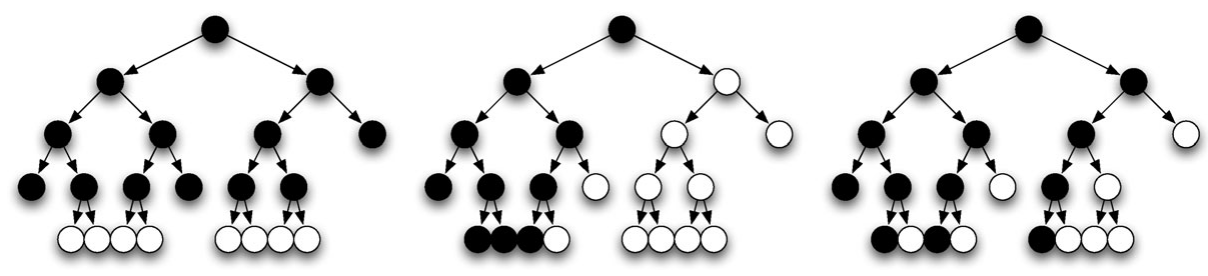

Fig. 11. The effect of the various search transformers on the search are shown: black nodes are visited, and white nodes are not. From left to right: depth bound 4, node bound 10, and limited discrepancy 1 .

$$
\begin{aligned}
& \mathrm{ts}=0=\text { continue } \mathrm{q} t \text { True } \\
& \mathrm{l} \text { otherwise }=\text { eval' tree q } \mathrm{t} \text { es } \mathrm{ts}
\end{aligned}
$$

The effect of the above three search transformers is sketched in Figure 11.

\subsection{Solver-dependent transformers}

The Transformer type class forces the transformer to be fully independent of the constraint solver used. However, in some cases we want the transformer to be aware of it. For instance, assume that the FD solver keeps track of the number of times constraints are woken up and reconsidered for propagation, which can be queried with wakeUps : : FD Int. Now we want a transformer that limits the overall number of wake-ups; it obviously has to be aware that the FD solver is being used to call the wakeUps function.

We can solve this problem by specifying what solver a transformer depends on:

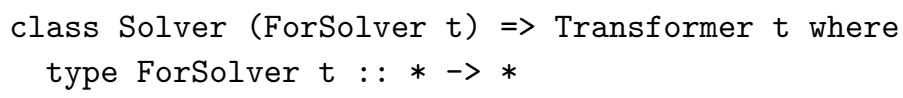

The above transformers of course keep on working for any solver, by adding the solver as a phantom type parameter, e.g.,

data DepthBoundedST solver $=$ DBST Int

instance (Solver solver) $\Rightarrow$ Transformer (DepthBoundedST solver) where

type ForSolver (DepthBoundedST solver) = solver

However, we can now also express the wake-up bounded transformer, which only works for the FD solver:

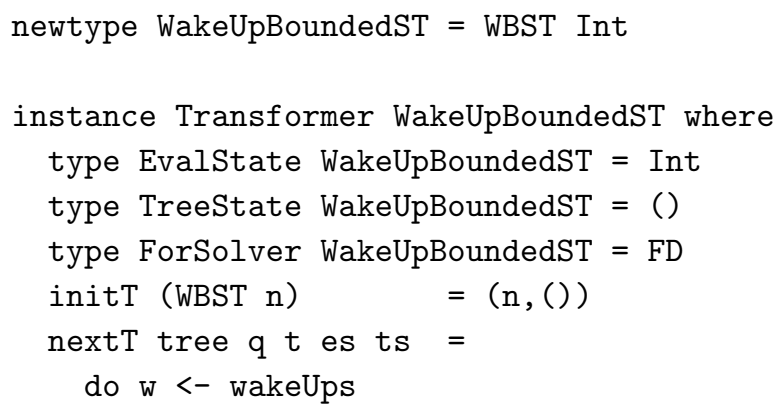




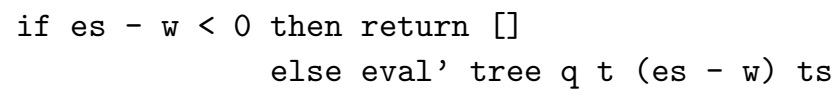

We'll see more fundamental uses of solver dependency later.

\subsection{Other transformers}

In the above, the transformer interacts with the evaluator when pushing and popping the queue. As an obvious extension of the same principle, the transformer can be made to interact at other points. We obtain a general transformer-evaluation interaction systematically if we replace each call to eval' and continue with a call to a distinct function in the Transformer type class.

Randomizing search transformer. An application of this is the randomizing search transformer, which is applied every time a Try node is evaluated. It randomly swaps the order of the two children of the Try node. The randomizing search transformer only makes use of the eval state that stores a randomly generated lazy list of Booleans indicating whether to swap the order of the next Try node or not:

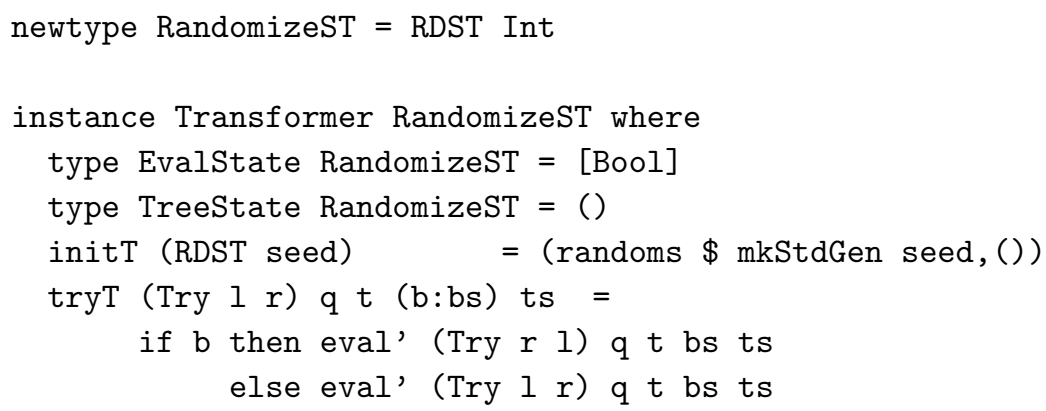

There are many other search transformers we can define in this manner, including finding only the first $k$ solutions, adding a counter to the search (e.g., to return the number of nodes explored), and changing the labeling strategies at some depth, among others.

\section{Composable search transformers}

We observe that many search transformers can conceptually be composed to obtain more complex and advanced ones. For instance, a combination of limiteddiscrepancy search and depth-bounded search, with well-chosen limits, realizes a different pruning of the tree than by either independently.

Unfortunately, the approach of the previous section does not support this compositional view at all: a conceptual composition of transformers means writing a new transformer from scratch. What we want is a plug-and-play solution, where we can easily compose complex search transformers from simple ones, maximizing reuse and programmer convenience. 
Composable transformer interface. Hence, we define a new type class CTransformer of composable transformers:

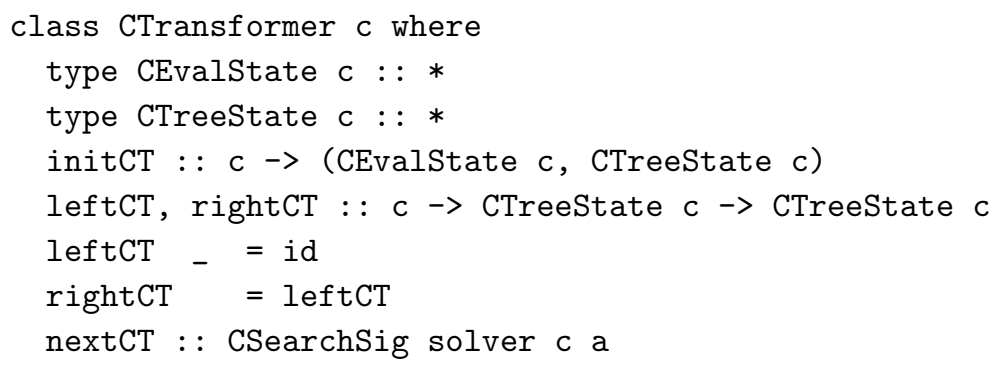

The interface of this type class is quite close to that of Transformer. The difference is hiding in the type of nextCT:

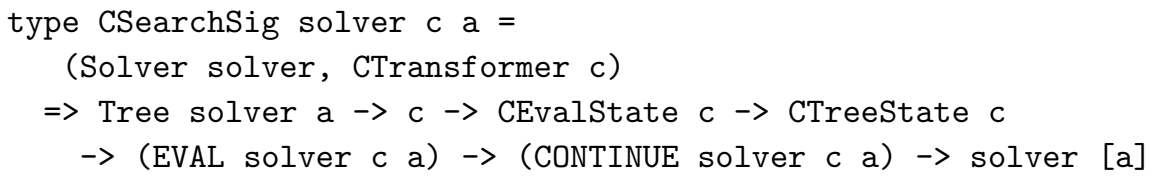

On the one hand, it is simpler than that of nextT because the queue type is no longer mentioned: at this point we do not expect the transformer to manipulate the queue.

On the other hand, it has two new parameters: EVAL solver $c$ a and CONTINUE solver $c$ a. These parameters are necessary for compositionality. As the transformer now does not (necessarily) run on top of a primitive search but (possibly) on top of a stack of other transformers, it is not allowed to call eval' and continue directly. Doing that would simply bypass all other transformers. Hence, EVAL solver $c$ a is an abstraction of eval' that takes care of the other transformers in the stack before calling the actual eval'; it is the same for CONTINUE solver $c$ a and continue:

type EVAL solver $\mathrm{c}$ a $=$ (Tree solver a $\rightarrow$ CEvalState $\mathrm{c} \rightarrow$ solver [a]) type CONTINUE solver $\mathrm{c}$ a $=$ (CEvalState $\mathrm{c} \rightarrow$ solver $[\mathrm{a}]$ )

One can think of these functions as continuations. The former proceeds with the search, while the latter aborts the search at the current location in the tree and continues with the next location in the queue.

Transformer composition. Composable transformers are composable because we can compose them into new composable transformers. In particular we compose them by stacking them.

We define the composition as a data type

data Composition es ts where

$(:-)$ : : (CTransformer a, CTransformer b)

$\Rightarrow \mathrm{a} \rightarrow \mathrm{b}$

$\rightarrow$ Composition (CEvalState a,CEvalState b) (CTreeState a,CTreeState b) 
that contains two composable transformers. The components, as existential types, are hidden; only their states are exposed.

The whole point of such a composition is that it is a composable transformer again:

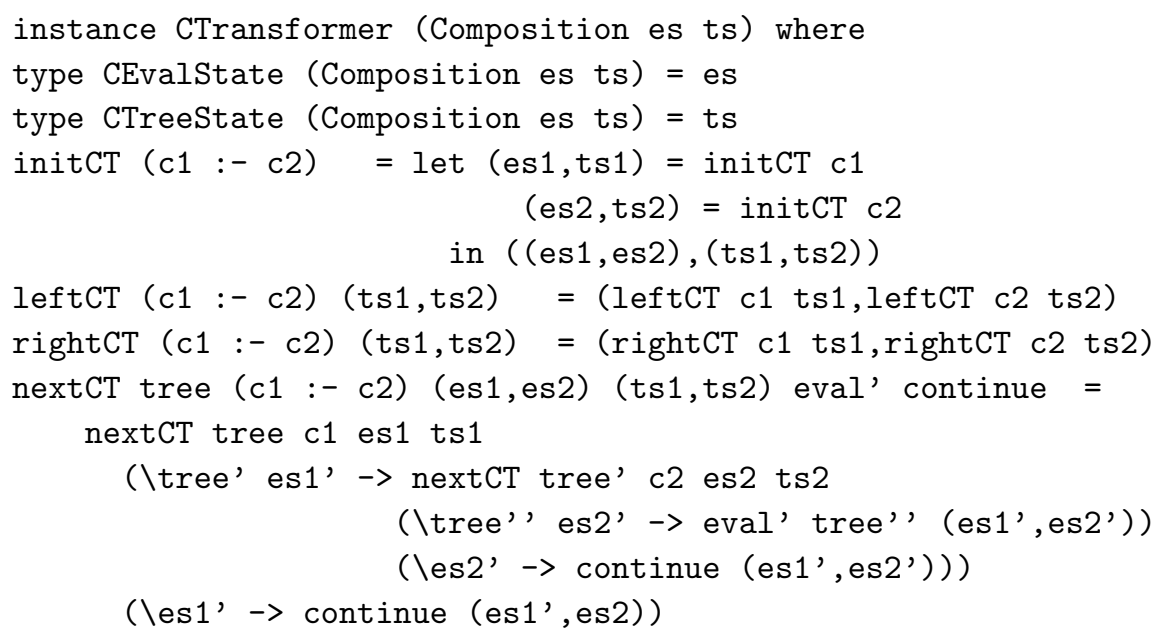

The above code is fairly straightforward. The function of interest is nextT, which in continuation passing style first calls the first component and then the next. Note that neither component needs to know about the other.

Composable transformers as transformers. Now, we can turn any composable transformer (usually a stack of composable transformers) into an ordinary transformer by means of the TStack transformer:

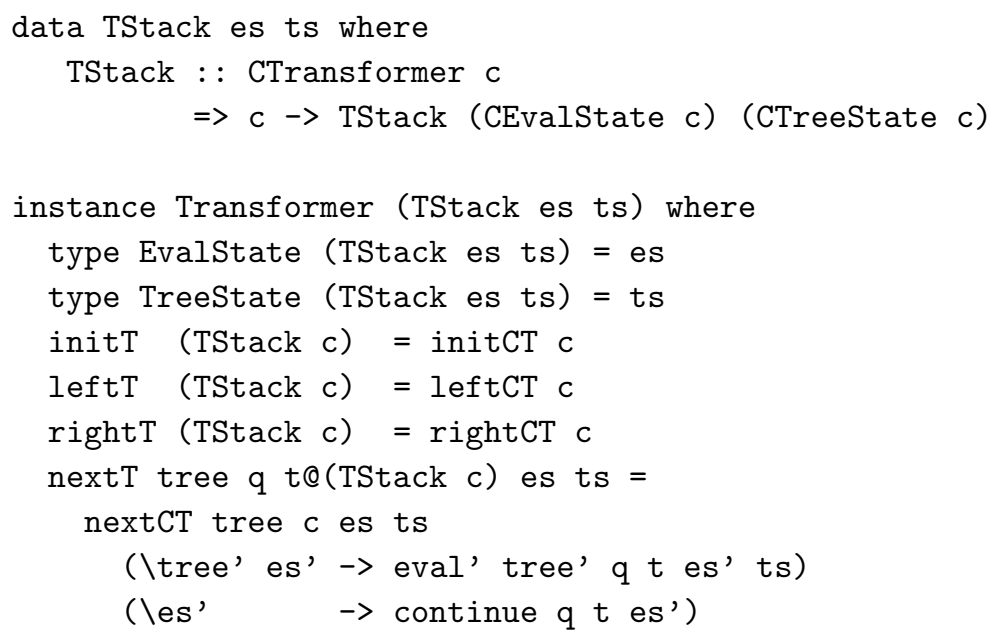

Here we see that the base continuations are indeed eval' and continue, as expected. 
Table 2. Composable transformer library

\begin{tabular}{ll}
\hline \hline Shorthand notation & Description \\
\hline it & identity transformer \\
db $n$ & depth bound $n$ \\
nd $n$ & node bound $n$ \\
ld $n$ & limited discrepancy $n$ \\
fs & first solution only \\
ra $n$ & randomizing with seed $n$ \\
bb $f$ & branch and bound with bound updater $f$ \\
\hline
\end{tabular}

\subsection{Composable transformer implementations}

The implementation of composable transformers is much the same as that of ordinary transformers. For instance, here is the adapted, now-composable, depthbounded transformer:

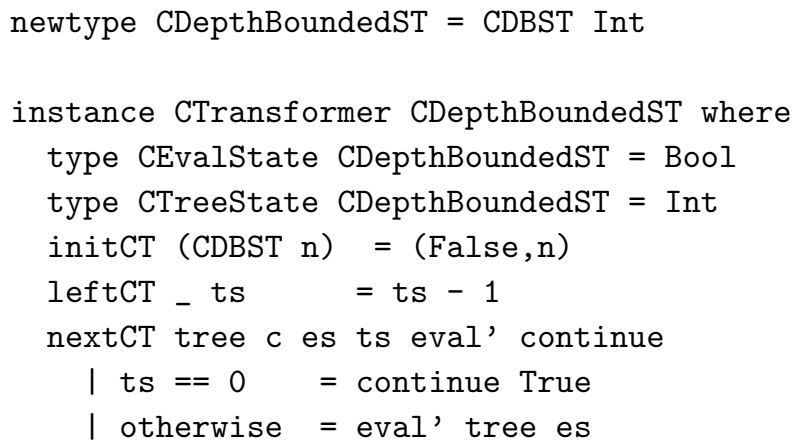

Combining this one with limit 40 with a composable node-bounded transformer with limit 20 and running the search is as easy as writing

$$
\text { solve model = run } \$ \text { eval model [] (TStack (CNBST } 20 \text { :- CDBST 40)) }
$$

We have implemented a small library of composable transformers, summarized in Table 2. With this library, we can simply plug and play and try out lots of different combinations.

For instance, we can experiment with the effect of different search strategies on finding the first solution of $n$ queens. Table 3 compares randomizing search (seed 13), limited discrepancy (with limit 10), and their composition on in order labeling. The results show that randomizing can improve a poor labeling, while LDS can be significantly worse, and combining them ameliorates the worst of the LDS. They also illustrate how the transformers do not commute; it's better to randomize before LDS than after.

\subsection{Branch-and-bound optimization}

The branch-and-bound search strategy is a classic approach to optimization. After it has found a solution, it is only interested in finding better solutions next. In other 
Table 3. Comparing the results of search transformers on default in order labeling for $n$ queens

\begin{tabular}{rrrrrrr}
\hline \hline$n$ & \multicolumn{1}{c}{ it } & ra 13 & ld 10 & ld 10 & :- ra 13 & ra $13:-$ ld 10 \\
\hline 1 & 8 & 8 & 8 & 8 & 8 \\
2 & 13 & 13 & 13 & 13 & 13 \\
3 & 23 & 23 & 23 & 23 & 23 \\
4 & 52 & 53 & 52 & 53 & 53 \\
5 & 62 & 62 & 62 & 62 & 62 \\
6 & 117 & 128 & 117 & 128 & 128 \\
7 & 107 & 107 & 107 & 107 & 107 \\
8 & 254 & 180 & 254 & 180 & 180 \\
9 & 190 & 201 & 190 & 201 & 201 \\
10 & 285 & 494 & 285 & 490 & 489 \\
11 & 261 & 266 & 261 & 266 & 266 \\
12 & 514 & 545 & 513 & 543 & 543 \\
13 & 370 & 486 & 370 & 486 & 486 \\
14 & 1,995 & 1,619 & 8,930 & 4,919 & 1,683 \\
15 & 1,555 & 3,183 & 1,366 & 1,120 & 1,104 \\
16 & 9,738 & 3,342 & 137,720 & 11,854 & 9,584 \\
17 & 6,430 & 6,430 & 3,759 & 6,640 & 4,931 \\
\hline \hline
\end{tabular}

words, it tries to prune the search tree by eliminating subtrees that do not yield a better solution.

The quality of a solution is based on an objective constraint variable. The value assigned to this variable determines the quality. Usually, the smaller the value, the better the solution.

We can easily add a generic branch-and-bound strategy as a composable transformer in our framework. This transformer is parametric in a NewBound solver action. This action should be called when a solution is found; it returns a function, Bound solver, for imposing constraints on further subtrees to only look for better solutions:

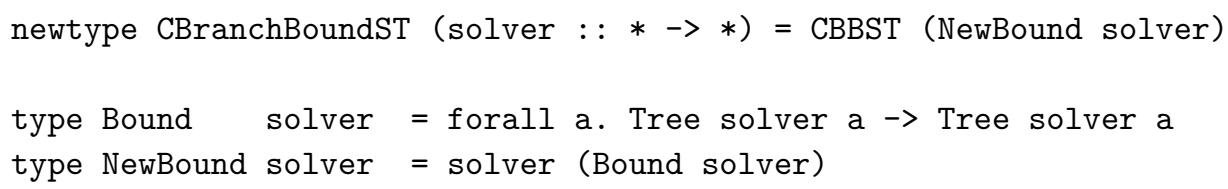

The transformer keeps track, in its evaluation state, of the above Bound solver function and applies it in nextCT. As an optimization, we want to apply each new function only once to any given subtree. Hence, the solver keeps track of the current function's version number in its evaluation state and of the version number of each subtree in its tree state:

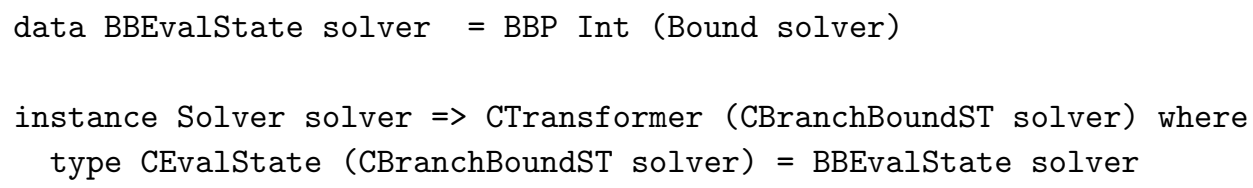




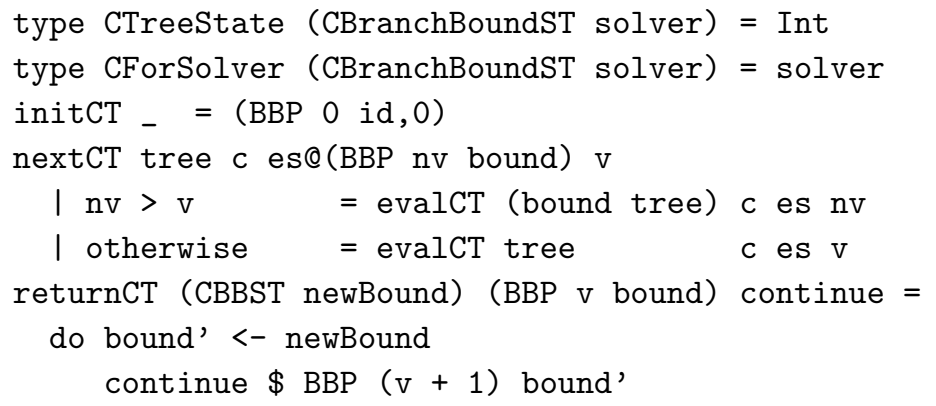

In the above, the returnCT function is an addition to the CTransformer type class, which allows interaction when a new solution is found. This function is used to obtain the new bounding function.

Note that Bound solver is a rank-2 type: it forces genericity in a, the result type of the whole computation.

Let us illustrate the branch-and-bound transformer for the FD solver with a small example. Assuming that objective : : FD FDVar returns the variable to be minimized, this is achieved by the following solver function:

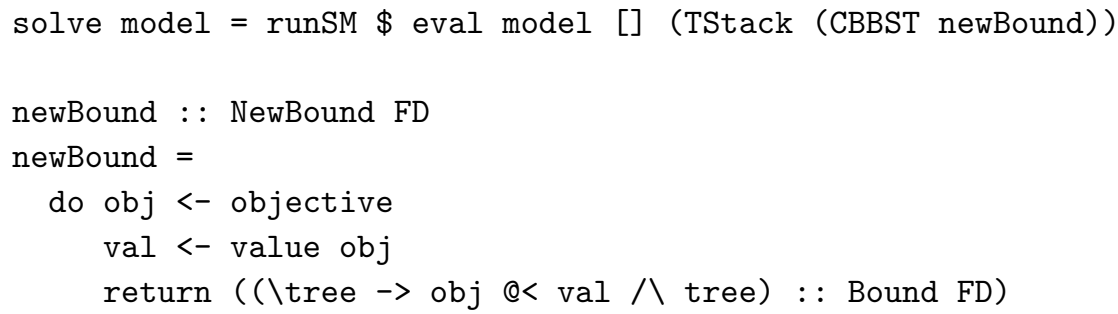

Whenever a new solution is found with objective value val, the tree transformer is changed to add a constraint demanding a better solution, i.e., with objective value smaller than val. This assumes that the variable to be minimized is fixed in any solution.

Here is a new twist on the traditional branch-and-bound algorithm. Optimistically, we assume that a solution can be found that is twice as good, i.e., whose objective value is less than half the current solution's objective value. Hence, we look for a new solution in the bottom half of the interval [0, val - 1] first. However, for completeness sake we also consider the upper half of that interval:

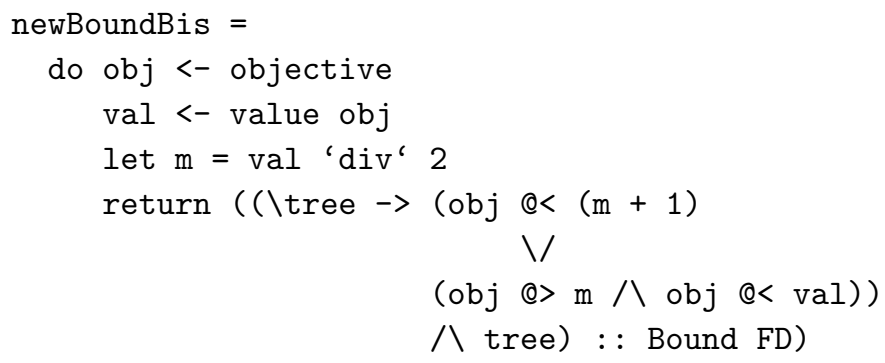

If the optimistic assumption is right a lot of the time, we make progress more quickly than in the previous approach. 


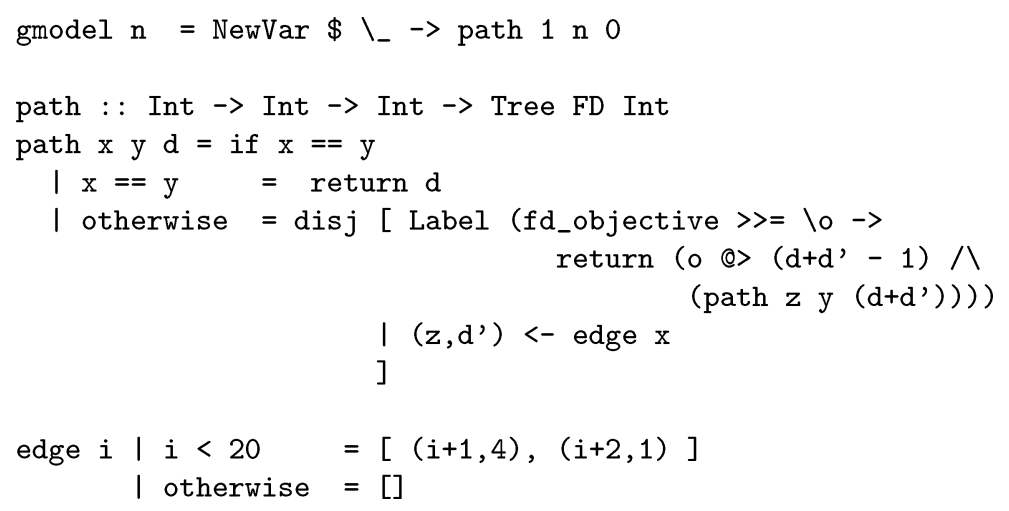

Fig. 12. Code to create a search tree for finding paths.

Note that this kind of optimization search, called optimistic partitioning, is commonly used in restart optimization, where we restart the search from scratch after each solution is found. But we are unaware of any literature that uses this transformation during search, while it's only a small change to our code.

Example 6. To illustrate branch-and-bound search we introduce a new example program. The code in Figure 12 defines a shortest path program. The solver only involves a single variable representing the path length, which is constrained by the labeling predicate path. A search tree is built by path based on edge $i$ that returns the list of nodes reachable from $i$ with their edge lengths.

The graph we use is a simple linear graph with edges from $i$ to $i+1$ of length 4 and to $i+2$ of length 1 (see Figure 13).

Table 4 compares the number of search tree nodes visited for different search strategies on the path program to find the best solution: without bounding (so looking for all solutions), using branch and bound and bisection branch and bound, and using BeFS.

BeFS differs in the queue data type from DFS: a priority queue maintains the subtrees to visit. A priority is assigned based on the (heuristic) likeliness of yielding a short path. For this purpose, we use the lower bound of the objective variable's domain, i.e., the distance so far. In other words, this BeFS realizes a greedy strategy, always extending the current shortest (partial) path.

We can see the significant improvements in using branch-and-bound search and how bisection branch-and-bound search improves upon this. Of course for these examples BeFS, which is an informed search strategy, is significantly better, but informed search strategies are usually not available in typical CP problems.

\subsection{Restarting transformer}

Some search strategies revisit a tree - usually different parts of it - multiple times. Two typical examples are iterative deepening and restart optimization. Iterative deepening repeatedly performs a depth-bounded search, each time increasing the 


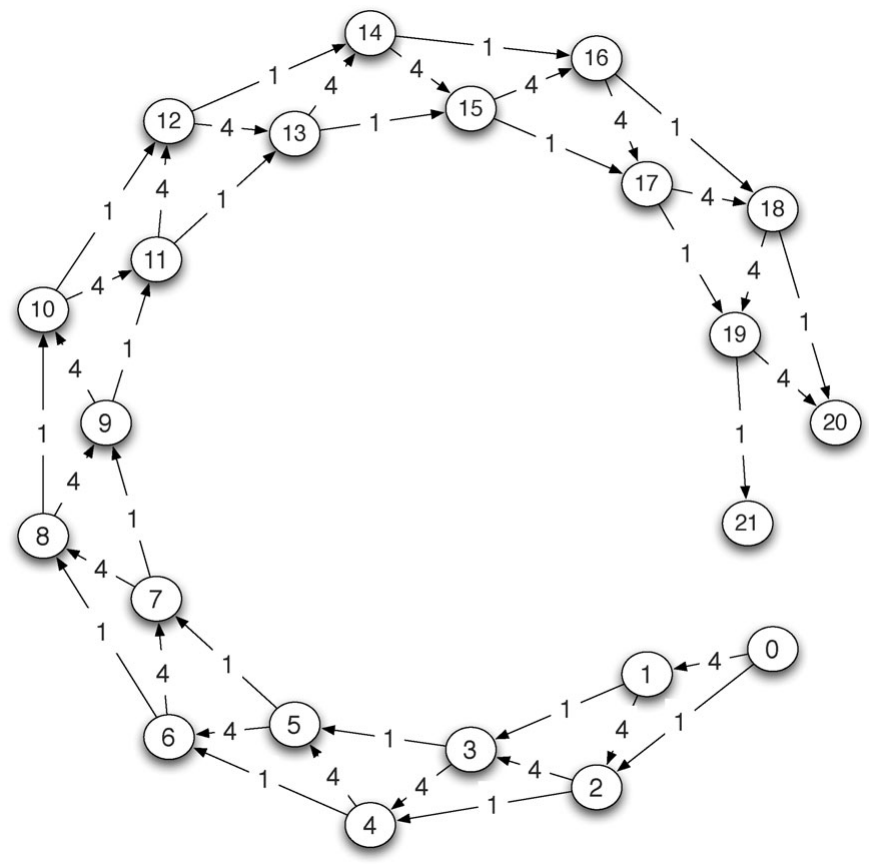

Fig. 13. Example graph for shortest path search.

depth limit. Restart optimization is similar to branch and bound but restarts from scratch: each time it tightens the bound on the objective variable using the previous solution found.

We propose the following generic RestartST transformer that captures the common pattern of iterative deepening and restart optimization. As we present this new transformer, we will introduce the necessary extensions of our framework as we go.

Firstly, the restart transfomer looks as follows:

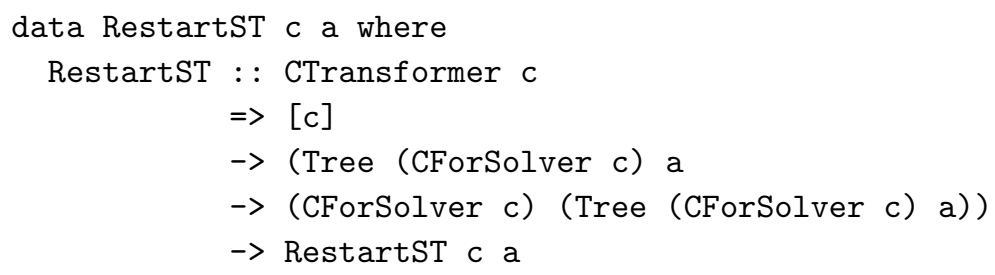

That is, it captures a sequence of composable transformers to iterate through and a function for updating trees.

The restart transformer is a regular transformer and not a composable one:

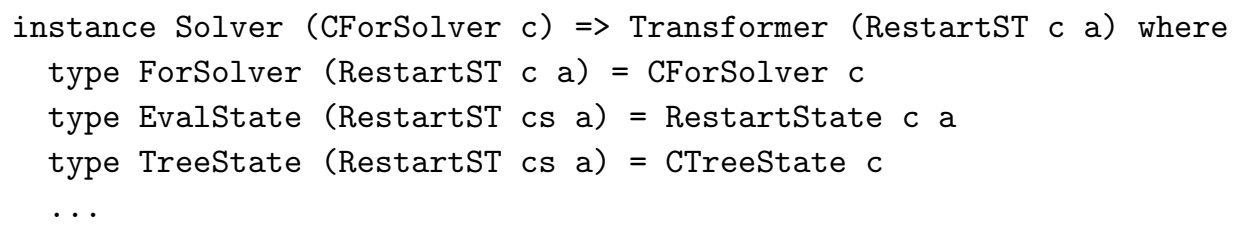


Table 4. Comparison of search strategies to find paths in the program shown in Figure 12 using the following: DFS finding all solutions (DFS ALL), DFS with branch and bound (DFS BB), DFS with bisection branch and bound (DFS BB-Bis), and BeFS (which requires the queueing strategy to be made aware of the distance so far)

\begin{tabular}{crrrr}
\hline \hline Destination & DFS ALL & DFS BB & DFS BB-Bis & BeFS FS \\
\hline 1 & 2 & 2 & 2 & 2 \\
2 & 33,450 & 45 & 52 & 5 \\
3 & 20,682 & 56 & 57 & 9 \\
4 & 25,570 & 110 & 110 & 13 \\
5 & 23,722 & 176 & 126 & 17 \\
6 & 24,458 & 252 & 197 & 21 \\
7 & 24,226 & 395 & 263 & 25 \\
8 & 24,394 & 504 & 337 & 29 \\
9 & 24,458 & 757 & 464 & 33 \\
10 & 24,642 & 910 & 716 & 37 \\
11 & 24,906 & 1,317 & 780 & 49 \\
12 & 25,354 & 1,525 & 1,147 & 53 \\
13 & 26,050 & 2,141 & 1,220 & 73 \\
14 & 27,242 & 2,426 & 1,777 & 77 \\
15 & 29,002 & 3,048 & 1,734 & 105 \\
16 & 32,290 & 3,433 & 2,487 & 109 \\
17 & 36,458 & 4,265 & 3,086 & 145 \\
18 & 46,218 & 4,793 & 3,425 & 149 \\
19 & 54,114 & 5,978 & 4,266 & 201 \\
20 & 87,562 & 6,704 & 4,718 & 205 \\
21 & 87,562 & 8,416 & 5,883 & 214 \\
\hline \hline
\end{tabular}

Most of the time, the restart transformer lets one of the captured transformers do its job. Hence, the tree state is that of $c$. The evaluation state is more involved:

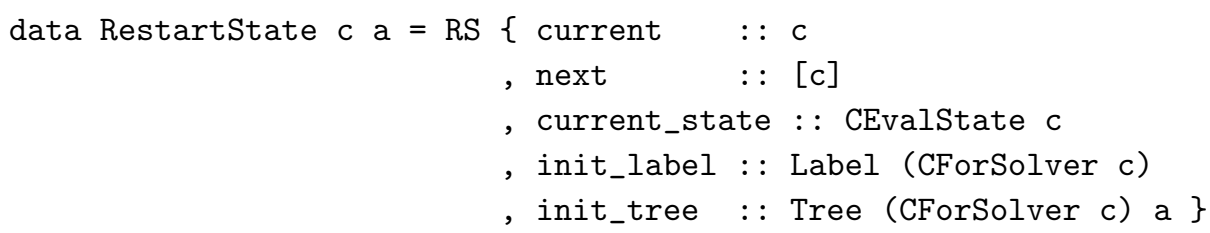

That is, it maintains the currently active composable transformer and its evaluation state, as well as the remaining composable transformers. The last two components are the initial model tree and the associated initial solver state. In order to allow for the initialization, we generalize the signature of initT to allow for the following implementation:

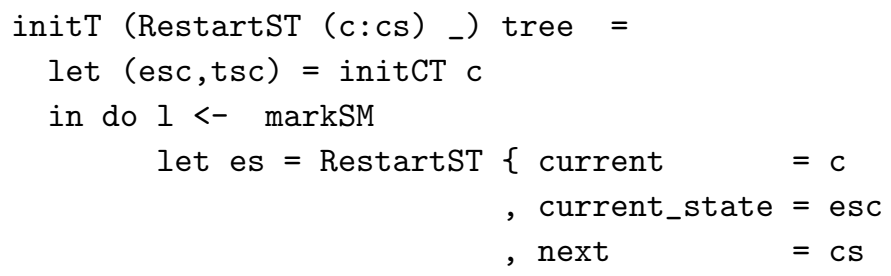


return (es, tsc)

$$
\begin{array}{ll}
\text { init_label } & =1 \\
\text { init_tree } & =\text { tree }
\end{array}
$$

Observe that as the type of the evaluation state now depends on a, the search's result type, the transformer now also depends on that type. We express this by adding an additional associated type family to the Transformer class:

$$
\text { type ForResult (RestartST c a) = a }
$$

We omit most of the method implementations, as these are simply delegated to current. However, there is one new transformer method endT that is called when the queue has run out of elements to process. This method allows the restart transformer to start the worklist anew with the initial tree:

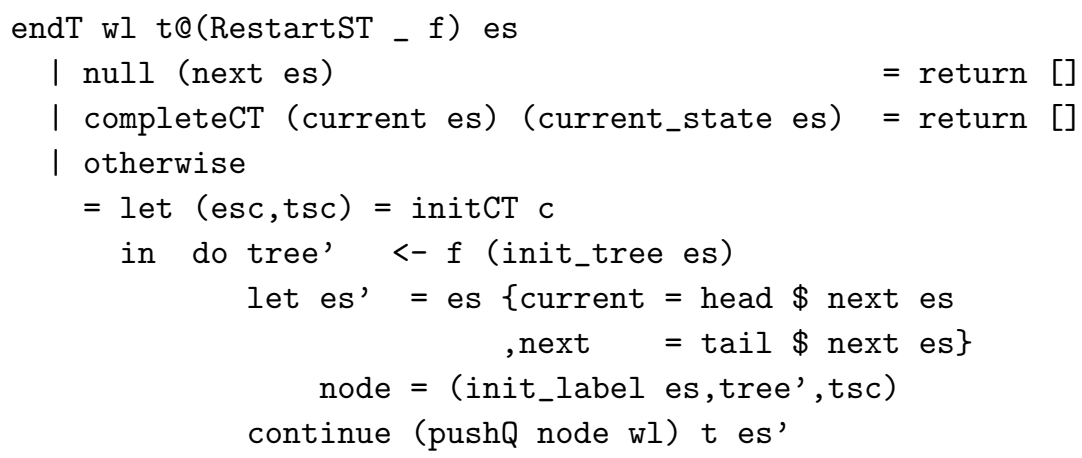

If there are no more composable transformers, then the search should end. Otherwise, if the last run has completely visited the tree, the search should end as well. We've added the completeCT member to the CTransformer type class. In all other cases, the initial tree is pushed onto the queue (after transformation by $f$ ), and the next composable transformer becomes the current one.

Now we can easily express iterative deepening as the restart optimization (RestartST (map db [1..]) return) and restart optimization as (RestartST (repeat $f s$ ) opt), where opt is defined as follows:

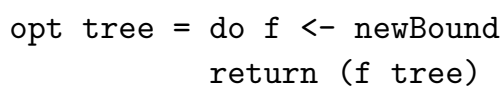

\subsection{Composable versus basic transformers}

As our framework provides both composable and basic search transformers, there may be a question of where to articulate a particular search strategy. Indeed, the same effect can be achieved in different ways. For instance, a combination of several composable transformers can also be implemented as a single monolithic basic search transformer.

In general, we would suggest to aim for increased flexibility. A composable search transformer is more readily reused as part of a different complex search strategy than a basic search transformer. The same holds for a basic search transformer 
and a primitive queue data structure versus a dedicate queue data structure with advanced behavior.

\section{Related work}

Since our approach combines constraint and functional programming there is a broad spectrum of related work.

CP. CLP languages such as ECLiPSe (ECLiPSe, 2008) and SICSTUS Prolog (SICStus, 2008) allow programmable search using the built-in search of the paradigm. Each system provides predicates to define search, analogous to the Dynamic nodes in the model tree. ECLIPSE provides a search library which allows user programmable variable and value selection (as in Section 5.2) as well as different search transformers including depth-bounded search, node-bounded search, and limited-discrepancy search, among others. One transformation cannot be applied to another, although one can change strategy, for example, when the depth bound finishes to another strategy. The user cannot define their own search transformers in the library, though they could be programmed from scratch.

The SALSA (Laburthe \& Caseau, 2002) language is an imperative domain-specific language for implementing search algorithms on top of constraint solvers. Its center of focus is a node in the search process. Programmers can write custom "Choice" strategies for generating next nodes from the current one; SALSA provides a regularexpression-like language for combining these Choices into more complex ones. In addition, SALSA allows custom procedures to be run at the exits of each node, i.e., right after visiting it. We believe that SALSA's Choice construct is orthogonal to our approach and could be easily incorporated. The custom exit procedures show similarity to our transformers, but no support is provided for composing transformers.

The Oz (Smolka, 1995) language was the first language to truly separate the definition of the disjunctive constraint model from the search strategy used to explore it (Schulte, 1997). Here computation spaces capture the solver state, as well as possible choices (effectively the Dynamic nodes). Search strategies such as DFS, BFS, LDS, branch and bound, and BeFS are constructed by copying the computation space and committing to one of the choices in the space. Search strategies themselves are monolithic; there is no notion of search transformers.

The original versions of the CP language OPL (Van Hentenryck, 1999; Van Hentenryck et al., 2000) provided a user programmable search language facility using a try construct, analogous to the Dynamic nodes in a model tree. The resulting tree could then be explored using a programmed exploration strategy (or built-in exploration strategies such as DFS, LDS, BFS, or BeFs). These explorations were based on a priority queue of nodes and programmed by giving a priority to each node, as well as a test to determine when to examine the next element in the queue rather than the children of the current node. This provided something equivalent to the Queue class. Exploration strategies could be modified by limit strategies that effectively created search transformers equivalent to our depth-bounded or nodebounded search transformers. These limit strategies appear to have been stackable. 
The work closest to this paper is the search language (Van Hentenryck \& Michel, 2006) of Comet (Van Hentenryck \& Michel, 2005). Search trees are specified using try and tryall constructs (analogous to Try and Dynamic nodes), but the actual exploration is delegated to a search controller that defines what to do when starting or ending a search, failing or adding a new choice. The representation of choices is by continuations rather than the more explicit tree representation we use. The SearchController class of Comet is roughly equivalent to the Transformer class. Complex search hybrids can be constructed by building search controllers. The Comet approach shares the same core idea as our monadic approach, to allow a threading of state through a complex traversal of the underlying search tree using functional abstractions and using that state to control the traversal. The Comet approach does not support a notion of composable search transformers. Interestingly the Comet approach to search can also be implemented in $\mathrm{C}++$ using macros and continuations (Michel et al., 2006).

Functional (constraint) logic programming. Several programming languages have been devoted to the integration of functional programming and (constraint) logic programming. On the one hand, we have CLP languages with support for a functional notation of predicates, such as Mercury (Somogyi et al., 1996) and Ciao (Casas et al., 2006). Mercury allows the user to program search strategies by using the underlying DFS, much like any CLP language. CIAO offers two alternative search strategies, BFS and iterative deepening, in terms of DFS by means of program transformation.

On the other hand, we have functional programming languages extended with logic programming features (nondeterminism, logical variables). The most prominent of these is the CURRY language or language family. The PACS CURRY compiler is implemented on top of SICSTUS PROLOG and naturally offers access to its constraint solver libraries; it has a fixed search strategy. However, the KICS CURRY system, implemented in HASKELL, does not offer any constraint solvers; yet, it does provide reflective access to the program's search tree (Brassel \& Huch, 2007), allowing programmed or encapsulated search. As far as we can tell, their implementation technique prevents this programmed search from being combined with constraint solving.

Embedding logic programming in functional programming. As far as we know, CP has gotten very little attention from mainstream functional programming researchers. Most effort has gone towards the study of the related domain of logic programming, whose built-in unification can be seen as an equality constraint solver for Herbrand terms.

There are two aspects to logic programming, which can be and have been studied either together or separately: logical variables and unification on the one hand and (backtracking) search on the other hand.

The former matter can be seen as providing an instance of a Herbrand term equality constraint solver for our Solver type class. However, it remains an open issue how to fit the works of Claessen and Ljunglöf (2000) and Jansson and Jeuring 
(1998) for adding additional type safety to solver terms into our solver-independent framework.

Logic programming and Prolog have also inspired work on search strategies in functional programming, which is to say, work on Prolog's dedicated search strategy: DFS with backtracking. Most notable is the list-based backtracking monad - which Wadler pioneered before the introduction of monads (Wadler, 1985) - upon which various improvements have been made, e.g., BFS (Seres \& Spivey, 1999), Prolog's pruning operator cut (Hinze, 2001), and fair interleaving (Kiselyov et al., 2005).

The Alma-0 (Apt et al., 1998) has a similar objective in an imperative setting: it adds Prolog-like DFS and pruning features to Modula-2.

FaciLe is a FD constraint library for OCaml, developed as part of the PhD thesis of Nicolas Barnier (2002). FaCiLe's fixed search stratgy is DFS; on top of this, optimization is possible by means of both the branch-and-bound and restart strategies. The implementation relies on mutable state.

In recent preliminary work, Fischer (2008) discussed how to add constraints to any instance of MonadPlus, with the goal of modeling functional logic programming in Haskell. In his approach, the search strategy is determined by the particular MonadPlus instance. There are no separate provisions for a queuing type or (composable) search strategy transformers.

Search in functional programming. Various specific instances of search-related problems have been solved in Haskell, of which the Sudoku puzzle is perhaps the most famous. While the Sudoku puzzle can be solved by many approaches, it is one at which FD CP excels: state-of-the-art FD solvers solve $9 \times 9$ puzzles in milliseconds. Yet, of the 19 Haskell Sudoku solvers currently on http:// haskell.org/haskellwiki/Sudoku, only one, by David Overton, considers an implementation in terms of an FD solver, and even that one implements a fixed DFS. Typical Haskell solutions, such as that of Bird (2006), implement problemspecific solvers with a hard-wired search strategy.

\section{Conclusion and future work}

We have given a monadic specification of $\mathrm{CP}$ in terms of a monadic constraint solver threaded through a monadic search tree. We show how the tree can be dynamically constructed through the so-called labeling methods and the order in which the nodes are visited controlled by a search strategy. The base search strategy can be transformed by search transformers, and indeed these can be constructed as composable transformations. Our framework allows the simple specification of complex search strategies, and illustrates how complex search strategies, like branch and bound, or iterative deepening can be built from smaller components. It also gives great freedom to explore new search strategies and transformers, for example, the optimistic branch-and-bound search.

Overall by trying to be as generic and modular as possible in defining monadic $\mathrm{CP}$ we have a powerful tool for experimentation and understanding of search in $\mathrm{CP}$. 


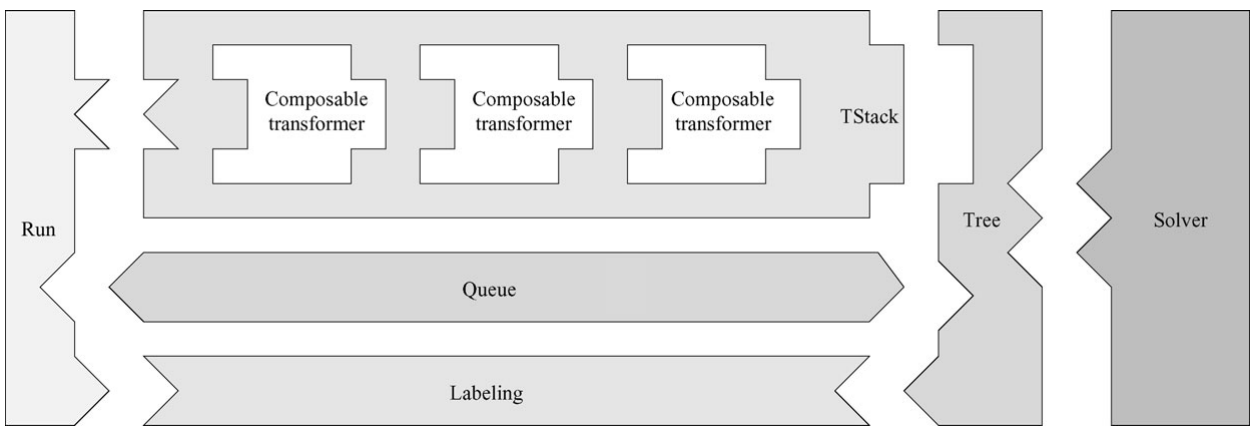

Fig. 14. The components of a CP model with composable search transformers and how they fit together.

The components, solver, search tree, labeling, queue, and search transformers are separate but nest together as shown in Figure 14.

In future work we would like to do the following:

- Generalize our search framework to arbitrary search problems.

- Integrate a Haskell implementation of constraint handling rules (Frühwirth, 1998) with the framework to provide the combination of programmable search and programmable solving.

- Make state-of-the-art constraint solver implementations, e.g., Gecode (Schulte et al., 2009), available, by binding them to Haskell using the $\mathrm{C}$ foreign function interface and have them implement the Solver type class.

- Investigate the connection between our composable search transformers and mixins (Cook, 1989), and in particular develop monadic mixins suitable for hiding the transformers' state.

- Explore the performance characteristics of the framework:

(1) the overhead of the search strategy transformers with respect to the basic search strategies and

(2) the overhead of the FFI bindings and search strategies with respect to native search strategies for state-of-the-art solvers.

\section{Acknowledgments}

We are grateful to the anonymous reviewers of this paper, whose comments and suggestions have significantly improved the presentation, as well as Ben Moseley, Serge Le Huitouze, Pieter Wuille, and Bruno Oliveira for their helpful comments.

\section{References}

Apt, K R., Brunekreef, J., Partington, V. \& Schaerf, A. (1998) Alma-o: An imperative language that supports declarative programming, ACM Trans. Program. Lang. Syst. 20 (5): 1014-1066.

Barnier, N. (Dec. 2002) Application de la programmation par contraintes à des problmes de gestion du trafic aérien, $\mathrm{PhD}$ thesis [online]. Toulouse, France: Institut National 
Polytechnique de Toulouse. Available at: http://www.recherche.enac.fr/opti/papers/ thesis/ Last accessed 3 August 2009.

Bird, R. S. (2006) A program to solve Sudoku, Journal of Functional Programming 16 (6): 671-679.

Brassel, B. \& Huch, F. (2007) On a tighter integration of Functional and Logic Programming. In The 5th Asian Symposium on Programming Languages and Systems (APLAS'07), Shao, Zhong (ed), Lecture Notes in Computer Science, vol. 4807. Springer, Berlin/Heidelberg, pp. $122-138$.

Casas, A., Cabeza, D., \& Hermenegildo, M. V. (2006) A syntactic approach to combining functional notation, lazy evaluation and higher-order in LP systems. In The 8th International Symposium on Functional and Logic Programming (FLOPS'06), Masami Hagiya and Philip Wadler (eds.), Springer, Berlin/Heidelberg, pp. 146-162.

Claessen, K., \& Ljunglöf, P. (2000) Typed logical variables in Haskell. In Proceedings of the Haskell Workshop, Montreal, Canada. ACM SIGPLAN.

Cook, W. R. (1989) A Denotational Semantics of Inheritance, PhD thesis. Providence, RI: Brown University.

ECLiPSe. (2008) Eclipse [online]. Available at: http://www.eclipse-clp.org/ Last accessed 3 August 2009.

Fischer, S. (2008) Constrained monadic computations [online]. Available at: http://www-ps . informatik.uni-kiel.de/ ${ }^{\sim}$ sebf/projects/constraint-monad.html Last accessed 3 August 2009.

Frühwirth, T. (1998) Theory and practice of Constraint Handling Rules, J. Logic Program. 37 (1-3): 95-138.

Hinze, R. (2001) Prolog's control constructs in a functional setting - axioms and implementation, Int. J Foundations Comp. Sci. 12 (2): 125-170.

Jaffar, J. \& Lassez, J.-L. (1987) Constraint logic programming. In POPL '87: Proceedings of the 14th ACM SIGACT-SIGPLAN Symposium on Principles of Programming Languages. Munich, Germany, pp. 111-119.

Jansson, P. \& Jeuring, J. (1998) Polytypic unification, J. Funct. Program. 8 (5): 527-536.

Kiselyov, O., chieh Shan, C., Friedman, D. P. \& Sabry, A. (2005) Backtracking, interleaving, and terminating monad transformers (functional pearl), SIGPLAN Not. 40 (9): 192-203.

Laburthe, F. \& Caseau, Y. (2002) SALSA: A language for search algorithms, Constraints 7 (3): $255-288$.

Marriott, K. \& Stuckey, P. J. (1998) Programming with Constraints: An Introduction. MIT Press, Cambridge, Massachusetts, USA.

Michel, L., See, A. \& Hentenryck, P Van. (2006) High-level nondeterministic abstractions in. In $C p$, Benhamou, F. (ed), Lecture Notes in Computer Science, vol. 4204. Springer, pp. 359-374.

Overton, D. (2008) Haskell FD library [online]. Available at: http://overtond.blogspot. com/2008/07/pre.html Last accessed 3 August 2009.

Régin, J.-C. (1994) A filtering algorithm for constraints of difference in csps. In Aaai, pp. 362-367.

Schrijvers, T., Peyton Jones, S., Chakravarty, M. \& Sulzmann, M. (2008) Type checking with open type functions. In Proceedings of the 13th ACM SIGPLAN international conference on Functional programming. ACM, pp. 51-62.

Schulte, C. (1997) Programming constraint inference engines. In Principles and Practice of Constraint Programming - cp97, Proceedings. Lecture Notes in Computer Science, vol. 1330. Springer, pp. 519-533. 
Schulte, C. (1999) Comparing trailing and copying for CP. In Proceedings of the 16th International Conference on Logic Programming, De Schreye, D. (ed), Las Cruces, NM: MIT Press, pp. 275-289.

Schulte, C., Duchier, D., Konvicka, F., Szokoli, G., Tack, G., Lagerkvist, M., Pekczynski, P., Reischuk, R. \& Guns, T. (2009) The Gecode generic constraint development environment [online]. Available at: http://www.gecode.org/ Last accessed 3 August 2009.

Seres, S. \& Spivey, M. J. (Sept. 1999) Embedding Prolog into Haskell. In Haskell Workshop '99. Paris, France.

SICStus. (2008) Sicstus Prolog [online]. Available at: http://www.sics.se/isl/sicstuswww/ site/index.html Last accessed 3 August 2009.

Smolka, G. (1995) The Oz programming model. In Computer Science Today, Lecture Notes in Computer Science, vol. 1000. Springer, pp. 324-343.

Somogyi, Z., Henderson, F. \& Conway, T. (1996) The execution algorithm of Mercury: An efficient purely declarative logic programming language, J. Logic Program. 29 (1-3): 17-64.

Van Hentenryck, P. (1999) The OPL Optimization Programming Language. Cambridge, MA: MIT Press.

Van Hentenryck, P. \& Michel, L. (2005) Constraint-Based Local Search. MIT Press.

Van Hentenryck, P. \& Michel, L. (2006) Nondeterministic control for hybrid search, Constraints 11 (4): 353-373.

Van Hentenryck, P., Perron, L., \& Puget, J.-F. (2000) Search and strategies in OPL, Acm Tocl. 1 (2): 285-315.

Wadler, P. (1985) How to replace failure by a list of successes, In Proceedings of a Conference on Functional Programming Languages and Computer Architecture. New York: Springer, pp. $113-128$. 